\title{
Fourier Transform for Paragroups and Its Application to the Depth Two Case
}

\author{
By
}

\author{
Nobuya SATO*
}

\begin{abstract}
We prove that the flatness condition in Ocneanu's paragroup theory for graphs with depth two is equivalent to existence of the multiplicative unitaries in the theory of Baaj-Skandalis by using "Fourier transform" introduced by A. Ocneanu. Moreover, from two Kac algebras dual to each other, we construct a subfactor as a crossed product by a Kac algebra action, with the string algebra construction.
\end{abstract}

\section{§1. Introduction}

Subfactor theory has explosively developed since its initiation by V.F.R. Jones and it has unexpectedly produced a similar structure to conformal field theory ([B-G]), 3-dimensional topological quantum field theory ([O4]), exactly solvable models $([\mathrm{R}])$, quantum groups $([\mathrm{W}])$, and so on. They have stimulated each other and subfactor theory has also enjoyed the effects.

In [Jo], V.F.R. Jones constructed subfactors of the approximately finite dimensional (AFD) factor of type $\mathrm{II}_{1}$ with all the possible index values. Later in 1987, A. Ocneanu announced a complete classification of AFD $\mathrm{II}_{1}$ subfactors with index less than four in [O1]. He used his original theory of paragroups [O1] for AFD $\mathrm{II}_{1}$ subfactors. For some time, his combinatorial theory had been mysterious, but the theory has been worked out by several people. The analytic aspects of the classification problem of subfactors have been fully completed by S. Popa in [P4].

The paragroup theory has very interesting aspects. One of them is that it has a (finite) group-like structure though they are based on infinite dimensional algebras, von Neumann algebras of type $\mathrm{II}_{1}$. If a subfactor arises as a crossed product by a finite group action, the paragroup for this subfactor contains the

Communicated by H. Araki, September 8, 1995.

1991 Mathematics Subject Classification: Primary 46L37.

*Department of Mathematical Sciences, University of Tokyo, 3-8-1 Komaba, Tokyo 153, JAPAN 
same group structure and also has the unitary representation theory of the original finite group. So paragroups seem to be "quantized groups" and general subfactors seem to be "paragroup crossed product" subfactors. This was the original motivation of Ocneanu for the initiation of the paragroup theory.

Among the axioms for paragroups, the flatness axiom is the most important. On one hand, if we have an irreducible inclusion of factors of type $\mathrm{II}_{1}$, we can construct a paragroup using bimodule theory (or correspondences [P2]) as in ([O3], [Y1]). On the other hand, if we have a bi-unitary connection (not necessarily flat), we can construct an inclusion of factors of type $\mathrm{II}_{1}$ by the string algebra construction as in [O3] and then the tower of the relative commutants of this inclusion is realized as the "flat part" of the string algebras [O3]. Moreover, if we have flatness, the inclusion constructed with string algebras has the same (dual) principal graphs as the original graphs. This fact was stated as the Range Theorem in [O1]. Flatness gives a compatibility for tensor products as bimodules as in [O4], [E-K1]. Moreover, it seems that flatness gives the above-stated group-like structures in irreducible inclusions of type $\mathrm{II}_{1}$ factors.

A. Ocneanu announced that an irreducible inclusion with depth two is described as a crossed product by a compact (or discrete) Kac algebra. Though several proofs have already been given (see $[\mathrm{Da}]$ and $[\mathrm{Sz}]$ for inclusions of type $\mathrm{II}_{1}$ factors with finite index, [Lo] for inclusions of properly infinite factors with finite index, and [E-N] for inclusions of properly infinite factors with infinite index), this theorem still has deep contents from the view point of group-like structures for paragroups.

In the present paper, we will study paragroups with two graphs with depth two and seek for the relation between flatness and group-like structures.

In section 2, we will review A. Ocneanu's paragroup theory.

In section 3, we will study Fourier transform for paragroups. This gives a powerful machinery to analyze the "group-like structure" of paragroups.

In section 4, we will prove our main theorem as follows. Assume there exists a bi-unitary connection with depth two. Then the flatness condition is equivalent to the existence of two multiplicative unitaries in the sense of Baaj-Skandalis ([B-S $]$ ).

In section 5, we will investigate the relation between the above two multiplicative unitaries.

In section 6 , we give a realization of a depth two paragroup from a Kac algebra. Moreover, it will be shown that a paragroup arising from a crossed product by an outer action of a Kac algebra is equal to the paragroup constructed with a Kac algebra. This suggests a relation to categorical aspects of paragroups (rigid monoidal tensor categories $[\mathrm{T}-\mathrm{V}],[\mathrm{Y} 2]$ ). 


\section{§2. Ocneanu's Paragroup Theory}

We review Ocneanu's paragroup theory to fix some notations. The present exposition is rather restricted. For general paragroups and more details, we refer readers to $[\mathrm{O} 1],[\mathrm{O} 3]$ and $[\mathrm{K}]$.

First, we have a graph $G$ consisting of four finite bipartite graphs $\mathscr{G}_{0}=\mathscr{G}_{3}$ $=\mathscr{G}, \mathscr{G}_{1}=\mathscr{G}_{2}=H$ as in the following figure.

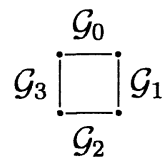

Suppose that $\mathscr{G}_{j}$ and $\mathscr{G}_{j+1}$ have common vertices $V_{j+1}$ and $\mathscr{G}_{j}$ 's have the common Perron-Frobenius eigenvalue $\beta$ and the common Perron-Frobenius eigenvector $\mu$ for $j \in \boldsymbol{Z} / 4 \boldsymbol{Z}$. We fix a vertex in $V_{0}$ (resp. $V_{2}$ ) called $*_{y}$ (resp. $\left.*_{\mathscr{H}}\right)$ and normalize $\mu$ so that $\mu(*)=1$. We call a combination of four edges, one from each graph, with common vertices, a call. We set one more assumption on the graphs (Initialization axiom) as follows.

There exists the only one vertex connected to $*_{g}\left(\right.$ resp. $\left.*_{\not 6}\right)$ in $V_{1}$ (resp. $V_{3}$ ) and it is the only vertex connected to $*_{\mathscr{H}}\left(\right.$ resp. $\left.*_{\mathscr{g}}\right)$ in $V_{1}$ (resp. $V_{3}$ ).

We assume that we have an assignment, called a connection, of a complex number to each cell and denote it $W$ and we use the graphical notation for the value of $W$ of a cell as in the following figure.

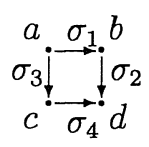

We set some assumptions on $W$ as follows (Unitarity axiom).

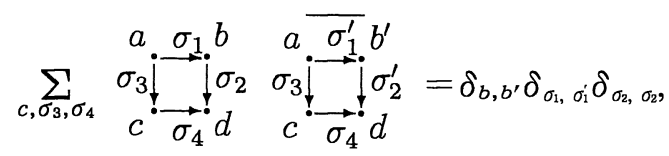

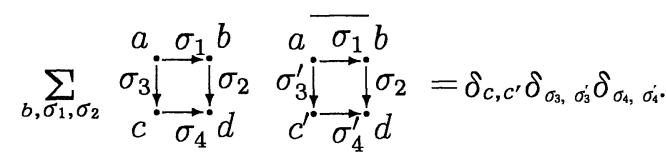

We can construct the nested graphs by reflecting each graphs vertically and horizontally. We use the notation $\sim$ to mean the reflected graphs and edges. We assume that $W$ on the nested graphs satisfies the following identity 
(Renormalization rule axiom).

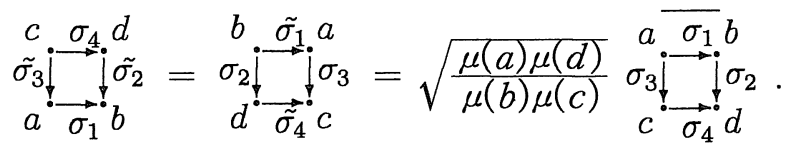

If a connection $W$ satisfies the above two conditions, we call it a bi-unitary connection.

For an oriented edge $\sigma$, we denote the starting point, the end point and the length by $s(\sigma), r(\sigma)$ and $|\sigma|$ respectively. We define an oriented path $\sigma$ on $\mathscr{G}_{0}$ by a succession of edges. We take a pair of paths $\left(\xi_{+}, \xi_{-}\right)$, called a string, which has the starting point $*$ and the same end point with length $n$.

First we construct an algebra $A_{0, n}$ from the above data.

Definition 2.1. We define $A_{0, n}$ as follows. As a $C$-vector space, a basis for $A_{0, n}$ is given by the strings with length $n$. The algebra structure is defined as follows. The product structure is given by $\left(\xi_{+}, \xi_{-}\right) \cdot\left(\eta_{+}, \eta_{-}\right)=\delta_{\xi_{-}, \eta_{+}}\left(\xi_{+}, \eta_{-}\right)$. The star-structure is given by $\left(\xi_{+}, \xi_{-}\right)^{*}=\left(\xi_{-}, \xi_{+}\right)$. Then $A_{0, n}$ is a finite dimensional $C^{*}$-algebra.

We can embed $A_{0, n}$ into $A_{0, n+1}$ canonically. Moreover there exists the unique normalized trace compatible with this embedding. Using this trace, we can construct an AFD II 1 factor $A_{0, \infty}={\overline{\bigcup_{n=1}^{\infty} A_{0, n}}}^{\text {weak }}$.

We can construct finite dimensional $C^{*}$-algebras $A_{k, n}$ on nested graphs in a similar way. Although we have many ways to reach at the $(k, n)$ component, the identification of different bases is given with the connection. We call these $A_{k, n}$ string algebras.

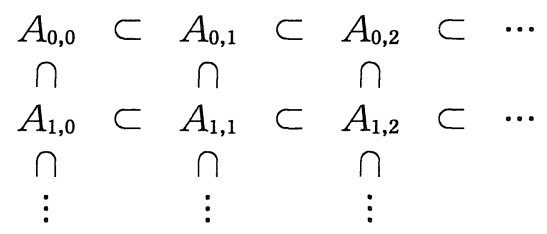

Thus we can construct increasing sequences of AFD $\mathrm{II}_{1}$ factors $A_{k, \infty}=$ $\cup_{n=0}^{\infty} A_{k, n}$ weak as well as $A_{0, n}$. We call this construction of AFD $\mathrm{II}_{1}$ factors the string algebra construction. We can also construct the string algebras $A_{-1, k}$ by identifying the one connected to $*_{y}$ and the edge connected to $*_{\not b}$ with Initialization axiom. We have the following theorem.

Theorem 2.2 ([03]). The inclusion $A_{0, \infty} \subset A_{1, \infty}$ is irreducible and the Jones index for this inclusion is given by $\beta^{2}$. The increasing sequence of $A F D$ $I I_{1}$ factors 


$$
A_{0, \infty} \subset A_{1, \infty} \subset A_{2, \infty} \subset A_{3, \infty} \subset A_{4, \infty} \cdots
$$

give the basic constructions for $A_{0, \infty} \subset A_{1, \infty}$. Moreover, we have an estimate $A_{0, \infty} \cap \cap A_{k, \infty} \subset A_{k, 0}$ for the higher relative commutants of $A_{0, \infty} \subset A_{1, \infty}$. Also we have an estimate $A_{-1, \infty}^{\prime} \cap A_{k, \infty} \subset A_{k, 1}$ for $A_{-1, \infty} \cap \cap A_{k, \infty}$.

Now we describe the most important axiom (Flatness axiom).

Definition 2.3 ( $[\mathrm{K}]$, Theorem 2.1). We say that a bi-unitary connection is flat if it satisfies the following equivalent conditions. Here $*$ means either $*_{9}$ or $*_{36}$.

1. Any two elements $x \in A_{k, 0}$ (in the vertical string algebra) and $y \in A_{0, l}$ (in the horizontal string algebra) commute.

2. For each horizontal string $\rho=\left(\rho_{+}, \rho_{-}\right) \in A_{0, k}$, we get the following identity, where $C_{\rho, \sigma} \in \boldsymbol{C}$ depends only on $\rho$ and $\sigma=\left(\sigma_{+}, \sigma_{-}\right)$.

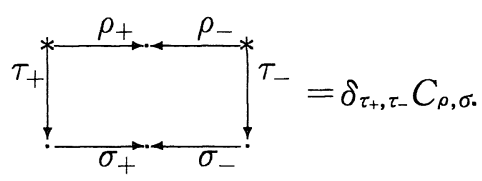

3. For any horizontal paths $\sigma_{+}, \sigma_{-}$and vertical paths $\rho_{+}, \rho_{-}$with all the sources and ranges equal to $*$, we get the following identity.

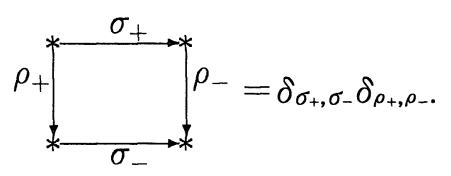

We explain the figures used above. First, reversed arrows define the new values as follows.

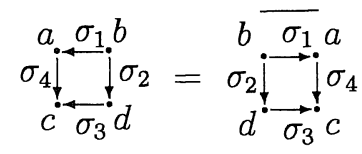

Next, the box-like figures mean the following. We make all the possible fillings of cells for the above diagrams. One such choice is called a configuration. We multiply the connection values of all the cells in a configuration and sum them over all the configurations. This is the value assigned to each of the above two box-like diagrams, and we mean this value by the diagram.

Definition 2.4. We call a bi-unitary flat connection $(G, W)$ a paragroup.

Theorem 2.2. and the following theorem give the reason the paragroup 
theory is important.

Theorem 2.5. (Popa's generating property [P3], [P4]) Let $N \subset M$ be an irreducible inclusion of AFD $I I_{1}$ factors with finite index and finite depth. Then we have the following anti-isomorphism.

$$
\left({\overline{\bigcup_{k=1}^{\infty} M_{1}^{\prime} \cap M_{k}}}^{\text {weak }} \subset{\overline{\bigcup_{k=1}^{\infty} M^{\prime} \cap M_{k}}}^{\text {weak }}\right) \cong(N \subset M) .
$$

Thus we can say as follows.

Theorem 2.6. A paragroup gives a complete invariant for irreducible inclusions of $A F D I I_{1}$ factors with finite index and finite depth.

\section{§3. Fourier Transform for Paragroups}

We freely use the notations in $[\mathrm{K}]$ and fix a paragroup $(G, W)$ with graphs $\mathscr{G}_{0}=\mathscr{G}_{3}=\mathscr{G}_{,} \mathscr{G}_{1}=\mathscr{G}_{2}=\mathscr{H}_{6}$ and denote the Perron-Frobenius eigenvalues for these graphs by $\beta$. Moreover, we note that we may change the connections by a gauge choice, which means a choice of an appropriate unitary operator, if necessary. So we choose the connection as follows.

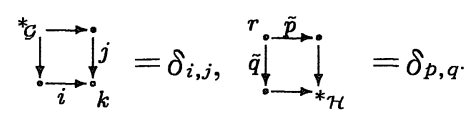

The meaning of the Kronecker $\delta$ on the right hand side is as follows. There is only one vertex on $\mathscr{G}$ that is connected to $*_{y}$ (resp. $*_{\mathscr{H}}$ ). For any $k$ (resp. $r$ ), the number of edges connecting such a vertex on $\mathscr{G}_{\text {and }} k$ (resp. $r$ ) on $\mathscr{G}_{1}$ (resp. $\mathscr{G}_{0}$ ) and that on $\mathscr{G}_{2}$ (resp. $\mathscr{G}_{3}$ ) are the same. By identifying these pairs of edges and denoting the above vertex simply by "•" (without any label), we can impose the above formula.

A. Ocneanu has defined Fourier transform for a paragroup first. Following [O2] and [O3], we define Fourier transform.

Definition 3.1. We define the linear map $\mathscr{F}: A_{0,2} \longrightarrow A_{1,1}$ by

$$
\mathscr{F}(x):=\beta^{3} E_{A_{1,1}}\left(x e_{0} e_{1}\right), x \in A_{0,2},
$$

and call this linear map $\mathscr{F}$ the Fourier transform for the paragroup.

We define the linear map $\tilde{F}: A_{1,1} \longrightarrow A_{0,2}$ as well by

$$
\widehat{\mathscr{F}}(x):=\beta^{3} E_{A_{0,2}}\left(x e_{1} e_{0}\right), x \in A_{1,1},
$$

and call this $\hat{\mathscr{F}}$ the inverse Fourier transform for the paragroup. Here $E$, 
$e_{0}$ and $e_{1}$ mean the conditional expectation and the two Jones projections, respectively $([K],[03])$.

Here we set some notations. We fix a system of matrix units $\left\{e_{i j}^{k}\right\}_{i, j=1, \cdots, n_{k}}$ ( $k^{\prime} s$ are odd vertices in the graph $\mathscr{G}_{2}$ ) in $A_{1,1}$ and another system of matrix units $\left\{\lambda_{p q}^{r}\right\}_{p, q=1, \cdots, n_{r}}\left(r^{\prime} s\right.$ are odd vertices in the graph ${\widetilde{G_{0}}}_{0})$ in $A_{0,2}$ for simplicity.

That is,

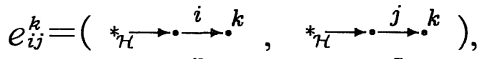

$$
\begin{aligned}
& \lambda_{p q}^{r}=\left({ }_{G}^{*} \longrightarrow \cdot \stackrel{p}{\longrightarrow} \bullet^{r},{ }^{*} \longrightarrow{ }_{\mathcal{G}} \longrightarrow \stackrel{q}{\longrightarrow}^{r}{ }^{r}\right) \text {. }
\end{aligned}
$$

Moreover, we use the notation $n_{k}=\mu(k)$ and $n_{r}=\mu(r)$.

Using the connection, the Fourier transform and inverse Fourier transform are expressed as follows.

\section{Proposition 3.2.}

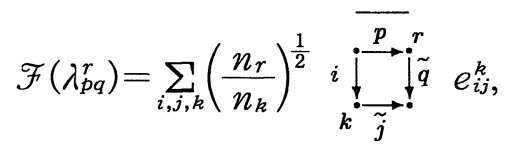

$$
\begin{aligned}
& \tilde{\mathcal{F}}\left(e_{i j}^{k}\right)=\sum_{p, q, r}\left(\frac{n_{k}}{n_{r}}\right)^{\frac{1}{2}} \underset{k}{i} \stackrel{p}{\stackrel{p}{\longrightarrow}} \stackrel{r}{j_{j}^{r}} !^{\tilde{q}} \lambda_{p q}^{r} .
\end{aligned}
$$

Proof. First we shall derive the string formula for $\lambda_{p q}^{r}$ in $A_{0,3}$.

$$
\lambda_{p q}^{r}=\left({ }_{\mathcal{G}} \longrightarrow \cdot \stackrel{p}{\longrightarrow} \cdot 0^{r}, \quad{ }^{*} \longrightarrow \cdot \stackrel{q}{\longrightarrow}^{*}{ }^{r}\right) \text { in } A_{0,2} .
$$

Imbed this string into $A_{0,3}$ and we get the following expression

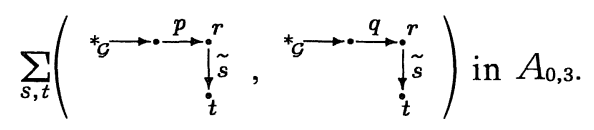

Using the connection, we identify this string naturally with the following expression

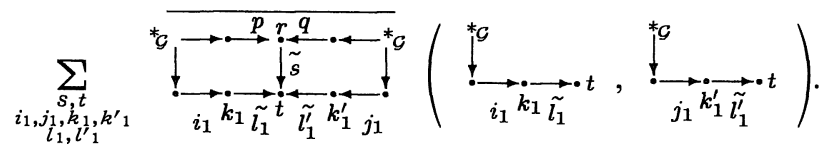

By Initialization axiom, we can identify the above equation with the following expression

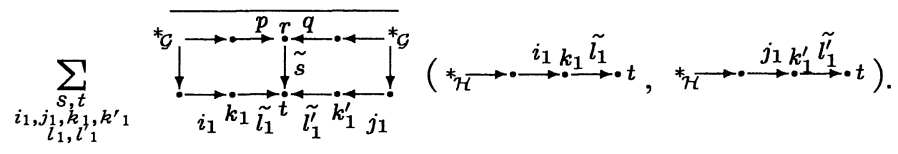


Next, we describe the Jones projections $e_{0}$ and $e_{1}$ graphically as follows.

$$
e_{0}=\left({ }^{*} \longrightarrow 0 \longrightarrow *_{\mathcal{H}},{ }^{*} \longrightarrow \cdot \longrightarrow *_{\mathcal{H}}\right) \text { in } A_{1,1} .
$$

We can imbed $e_{0}$ into $A_{1,2}$ and get the following expression.

$$
e_{0}=\sum_{t_{1}, l_{2}}\left({ }^{*} \longrightarrow \cdot \longrightarrow * \frac{l_{2}}{\mathcal{H}} \cdot t_{1},{ }^{*} \longrightarrow \cdot \longrightarrow * \frac{l_{2}}{\mathcal{H}} \cdot t_{1}\right)
$$

Similarly we have

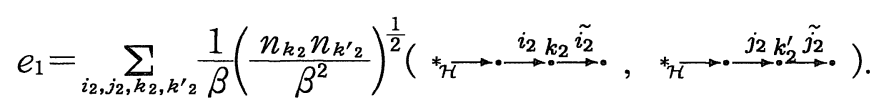

We can compute $\lambda_{p q}^{r} e_{0} e_{1}$ as follows.

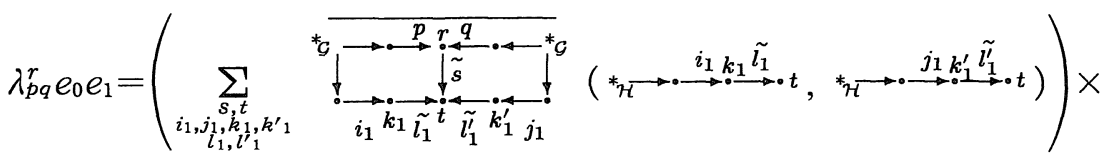

$$
\begin{aligned}
& \left(\sum_{t_{1}, l_{2}}\left({ }^{*} \longrightarrow \cdot \cdots * \frac{l_{2}}{\not{H}} \cdot t_{1},{ }^{*} \longrightarrow \cdot \longrightarrow * \frac{l_{2}}{\mathcal{H}} \cdot t_{1}\right)\right) \times
\end{aligned}
$$

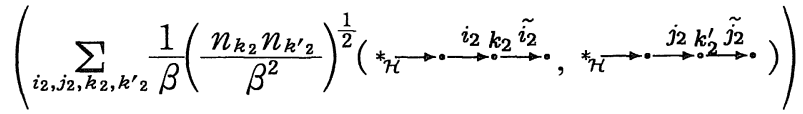

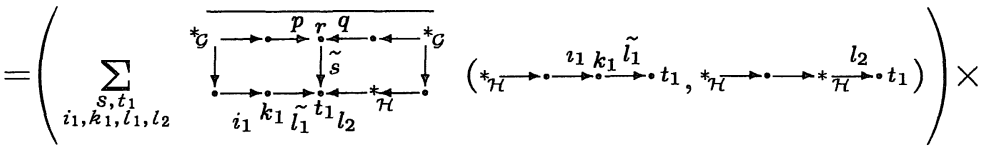

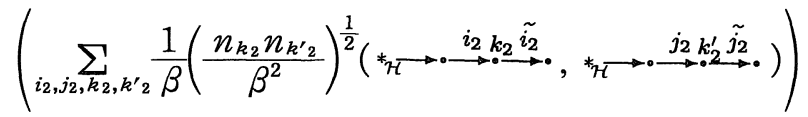

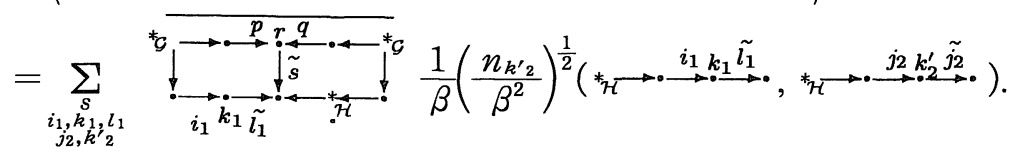

Applying the conditional expectation $E_{A_{1,1}}$ to this equation, we get

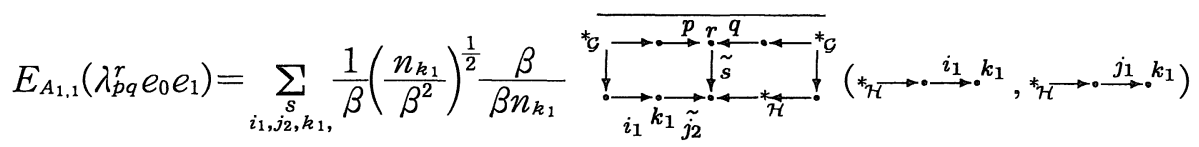

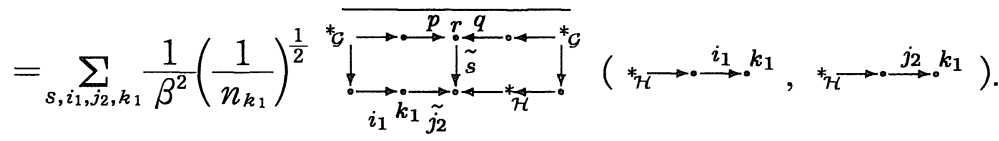

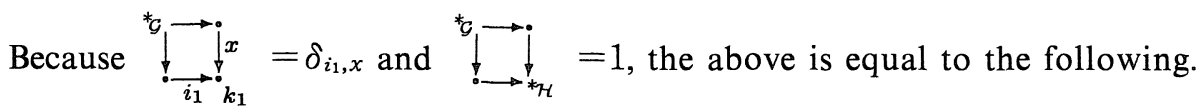




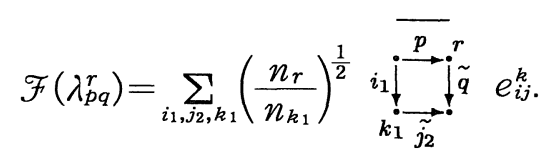

Thus we get the formula for the Fourier transform. We can deduce the formula for the inverse Fourier transform in a similar way.

Q. E. D.

Remark. In the case of a paragroup coming from an irreducible inclusion of type $\mathrm{II}_{1}$ factors $N \subset M$, we can easily check that $\left\{\left(\frac{n}{n_{r}}\right)^{\frac{1}{2}} \lambda_{p q}^{r}\right\}$ (resp. $\left\{\left(\frac{n}{n_{k}}\right)^{\frac{1}{2}}\right.$ $\left.\left.e_{i j}^{k}\right\}\right)$ gives a Pimsner-Popa basis for the inclusion $M_{1} \subset M_{2}$ (resp. $M \subset M_{1}$ ). So the above formulae are unitary transformations between the Pimsner-Popa bases for $N^{\prime} \cap M_{1}$ and $M^{\prime} \cap M_{2}$.

Proposition 3.3. The Fourier transform $\mathcal{F}$ is invertible and its inverse is the inverse Fourier transform $\hat{\mathscr{F}}$.

Proof. We shall check this by direct computation.

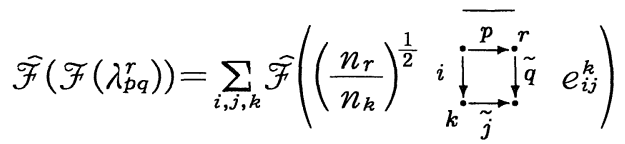

$$
\begin{aligned}
& =\sum_{i, j, k}\left(\frac{n_{r}}{n_{k}}\right)^{\frac{1}{2}} \underset{i}{\stackrel{\prod_{\vec{j}}}{\stackrel{p}{\longrightarrow}} !^{r} \tilde{q}} \tilde{\mathscr{F}}\left(e_{i j}^{k}\right)
\end{aligned}
$$

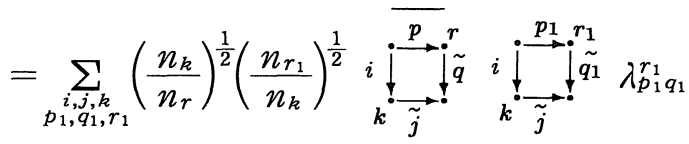

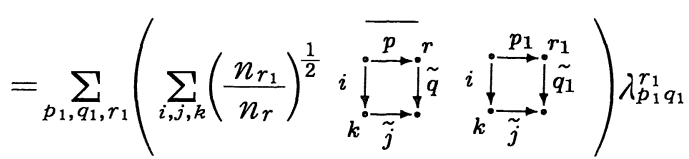

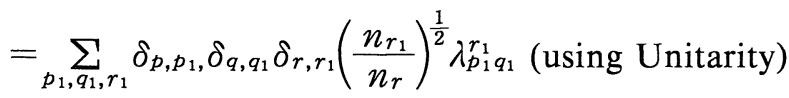

$$
\begin{aligned}
& =\lambda_{p q}^{r} \text {. }
\end{aligned}
$$

Similarly, we can compute the other as follows.

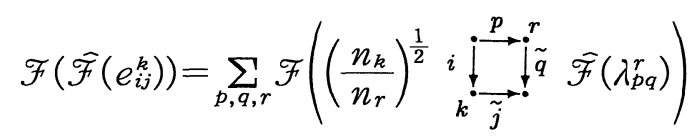




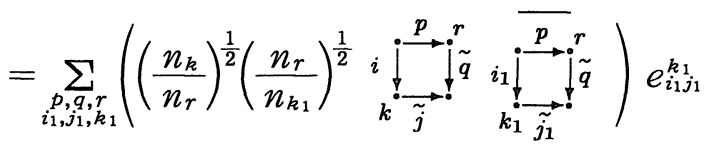

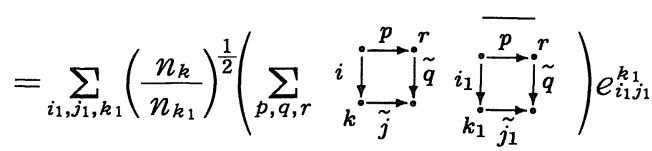

$$
\begin{aligned}
& =\sum_{i_{1}, j_{1}, k_{1}}\left(\frac{n_{k}}{n_{k_{1}}}\right)^{\frac{1}{2}} \delta_{i, i_{1}} \delta_{j, j_{1}} \delta_{k, k_{1}} e_{i_{1} j_{1}}^{k_{1}} \text { (using Unitarity) } \\
& =e_{i j}^{k} \text {. }
\end{aligned}
$$

This completes the proof.

Q. E. D.

Lemma 3.4. The Fourier transform and the inverse Fourier transform preserve the inner products arising from tr. That is, we have the following identities.

(i) $\quad(\mathscr{F}(x), \mathscr{F}(y))=(x, y), x, y \in A_{0,2}$.

(ii) $\quad(\hat{\mathcal{F}}(x), \overrightarrow{\mathcal{F}}(y))=(x, y), x, y \in A_{1,1}$.

Proof.

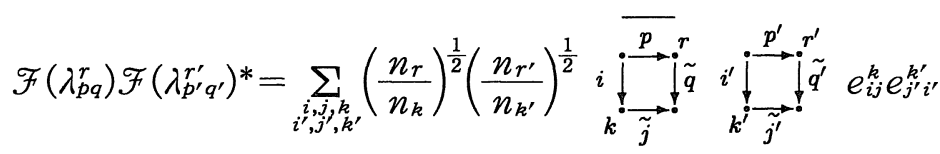

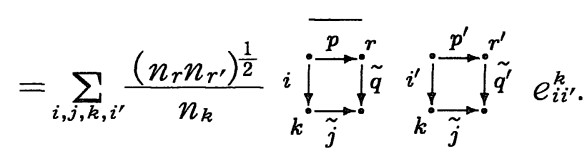

Applying the trace to this identity, we get

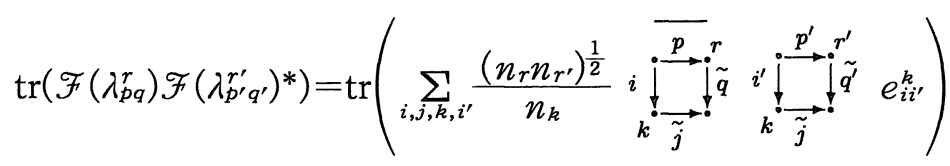

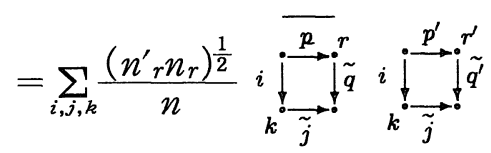

$$
\begin{aligned}
& =\delta_{p, p^{\prime}} \delta_{q, q^{\prime}} \delta_{r, r} \frac{n_{r}}{n} \text {. (using Unitarity) }
\end{aligned}
$$

On the other hand, we have

$$
\operatorname{tr}\left(\lambda_{p q}^{r} \lambda_{p^{\prime} q^{\prime}}^{r^{\prime}}{ }^{*}\right)=\delta_{q, q^{\prime}} \delta_{r, r^{\prime}} \operatorname{tr}\left(\lambda_{p p^{\prime}}^{r}\right)
$$




$$
=\delta_{p, p^{\prime}} \delta_{q, q^{\prime}} \delta_{r, r} \frac{n_{r}}{n}
$$

This completes the proof of (i).

By a similar computation, we can prove (ii).

Q. E. D.

By the above two Propositions, we get the following Proposition.

Proposition 3.5. The linear maps $\mathscr{F}$ and $\hat{\mathscr{F}}$ are unitary. and $A_{1,1}$.

Next, we introduce another product called the convolution product in $A_{0,2}$

Definition 3.6. We define the following new product in $A_{1,1}$.

$$
x * y:=\mathscr{F}(\hat{\mathscr{F}}(x) \hat{\mathscr{F}}(y)), x, y \in A_{1,1} .
$$

We also define the following new product in $A_{0,2}$.

$$
x \hat{*} y:=\hat{F}(\mathscr{F}(x) \mathscr{F}(y)), x, y \in A_{0,2} .
$$

Because $\mathscr{F}$ and $\hat{F}$ are the inverses of each other, we get the following identity.

$$
\begin{aligned}
& \hat{\mathscr{F}}(x * y)=\hat{\mathscr{F}}(x) \tilde{\mathscr{F}}(y), x, y \in A_{1,1}, \\
& \mathscr{F}(x \hat{*} y)=\mathscr{F}(x) \mathscr{F}(y), x, y \in A_{0,2} .
\end{aligned}
$$

Furthermore, we get the following Proposition by definition.

Proposition 3.7. The convolution products $*$ and $\hat{*}$ are associative.

Using the connection, we can describe the convolution products explicitly as follows.

Proposition 3.8. We get the following formulae for the convolution products.

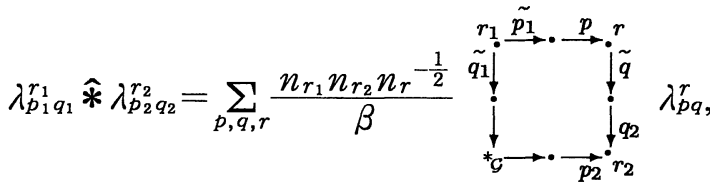

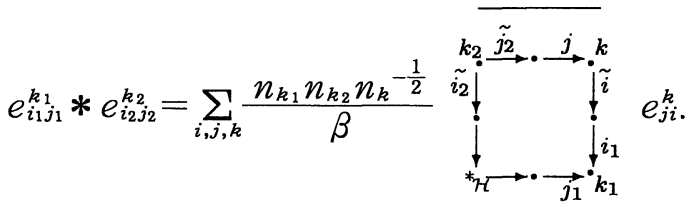


Proof. For (3.9), we can compute as follows.

$$
\begin{aligned}
& \lambda_{p_{1} q_{1}}^{r_{1}} \hat{*} \lambda_{p_{2} q_{2}}^{r_{2}}=\hat{\mathscr{F}}\left(\mathscr{F}\left(\lambda_{p_{1} q_{1}}^{r_{1}}\right) \mathcal{F}\left(\lambda_{p_{2} q_{2}}^{r_{2}}\right)\right)
\end{aligned}
$$

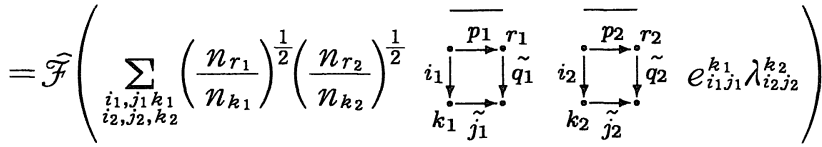

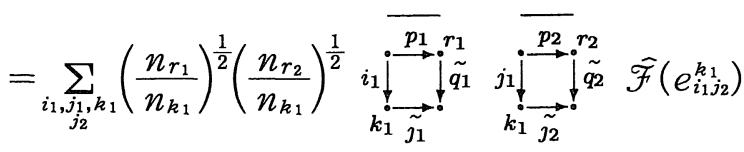

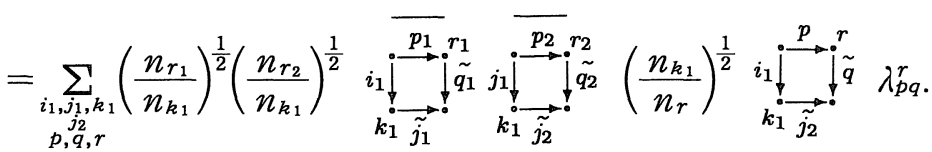

Using Renormalization rule for the first two connections, we get

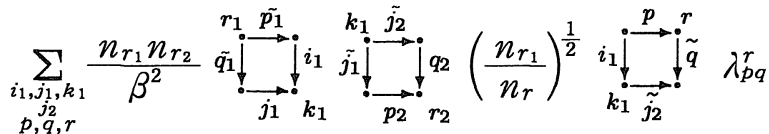

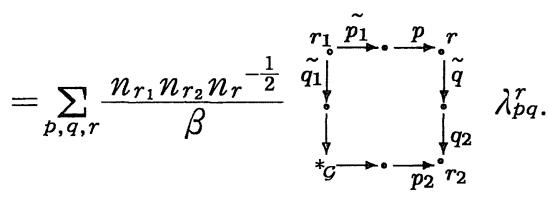

For (3.10), we can compute as follows.

$$
\begin{aligned}
& e_{i_{1} j_{1}}^{k_{1}} * e_{i_{2} j_{2}}^{k_{2}}=\mathscr{F}\left(\hat{\mathcal{F}}\left(e_{i_{1} j_{1}}^{k_{1}}\right) \hat{\mathscr{F}}\left(e_{i_{2} j_{2}}^{k_{2}}\right)\right)
\end{aligned}
$$

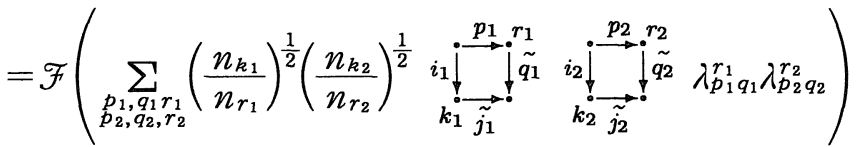

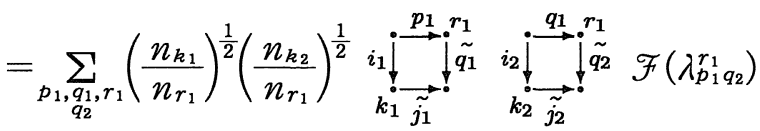

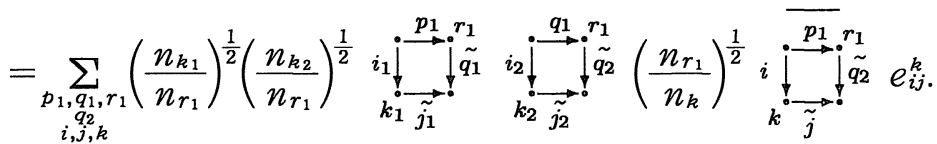

Using Renormalization rule for the first two connections, we get

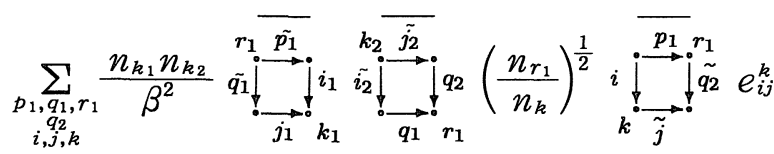




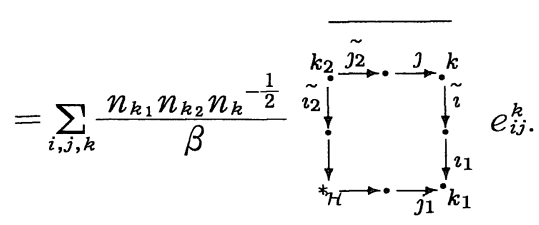

Q. E. D.

Definition 3.9. We define another star structure \# in $A_{1,1}$ as follows.

$$
x^{*}=\mathscr{F}\left(\hat{\mathcal{F}}(x)^{*}\right), x \in A_{1,1} .
$$

We define another star structure $\#$ in $A_{0,2}$ as follows.

$$
x^{\#}=\hat{\mathscr{F}}\left(\mathscr{F}(x)^{*}\right), x \in A_{0,2} .
$$

Thus we have the following property by definition.

$$
\begin{aligned}
& \hat{\mathscr{F}}\left(x^{\#}\right)=\hat{\mathscr{F}}(x)^{*}, \\
& \mathscr{F}\left(x^{\tilde{\#}}\right)=\mathscr{F}(x)^{*} .
\end{aligned}
$$

Proposition 3.10. The two algebras $\left(A_{1,1}\right.$, the convolution product $*$, the star structure \#) and $\left(A_{0,2}\right.$, the convolution product $\hat{*}$, the star structure $\left.\mathbb{\#}\right)$ are finite dimensional $C^{*}$-algebras.

So we can decompose these $\mathrm{C}^{*}$-algebras into direct sums of full matrix algebras as follows.

$$
A_{1,1}=\bigoplus_{r=1}^{s} M_{n_{r}}(\mathbf{C})
$$

We define a system of matrix units $\left\{\hat{\lambda}_{p q}^{r}\right\}_{p, q=1, \cdots, n_{r}}(r=1, \ldots, s)$ by $\hat{\lambda}_{p q}^{r}=$ $\mathscr{F}\left(\lambda_{p q}^{r}\right)$.

$$
A_{0,2}=\bigoplus_{k=1}^{l} M_{n_{k}}(\mathbf{C})
$$
$\widehat{\mathscr{F}}\left(e_{i j}^{k}\right)$.

We define a system of matrix units $\left\{\hat{e}_{i j}^{k}\right\}_{i, j=1, \cdots, n_{k}}(k=1, \ldots, l)$ by $\hat{e}_{i j}^{k}=$

Thus we have two finite dimensional $\mathrm{C}^{*}$-algebra structures in each of $A_{0,2}$ and $A_{1,1}$. We will see in the next section that these two algebra structures give a Kac algebra structure on each algebra.

\section{§4. The Relation between the Flatness Condition and the Pentagonal Identities for the Depth Two Case}

We apply all the results in the previous section to the depth two case and 
prove the main theorem in this section.

We need some preparations for proving the main theorem. We adopt the notation $n=\beta^{2}$.

Definition 4.1. Define linear functionals $\hat{\varphi}, \varphi$ as follows.

$$
\begin{aligned}
& \hat{\varphi}(x)=\beta \operatorname{tr}(x), x \in A_{0,2}, \\
& \varphi(x)=\beta \operatorname{tr}(x), x \in A_{1,1} .
\end{aligned}
$$

Definition 4.2. We define the linear maps $\hat{\Gamma}$ from $A_{0,2}$ to $A_{0,2} \otimes A_{0,2}$ and $\Gamma$ from $A_{1,1}$ to $A_{1,1} \otimes A_{1,1}$ as follows.

$$
\begin{gathered}
(\hat{\varphi} \otimes \widehat{\varphi})(\hat{\Gamma}(x)(a \otimes b))=\hat{\varphi}(x(a \hat{*} b)), x, a, b \in A_{0,2}, \\
(\varphi \otimes \varphi)(\Gamma(x)(a \otimes b))=\varphi(x(a * b)), x, a, b \in A_{1,1} .
\end{gathered}
$$

Proposition 4.3. The linear maps $\hat{\Gamma}$ and $\Gamma$ are described with the connection as follows.

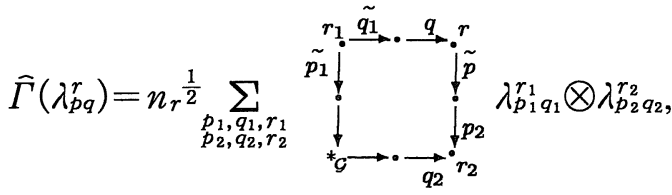

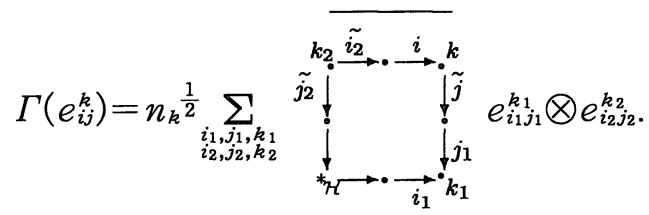

Proof. We shall prove only the formula for $\hat{\Gamma}$ because the formula for $\Gamma$ can be proved in a similar way.

We set

$$
\hat{\Gamma}\left(\lambda_{p q}^{r}\right)=\sum_{\substack{p_{1}, q_{1}, r_{1} \\ p_{2}, q_{2}, r_{2}}} C_{p_{1} q_{1} r_{1} p_{2} q_{2} r_{2}}^{p q r} \lambda_{p_{1} q_{1}}^{r_{1}} \otimes \lambda_{p_{2} q_{2}}^{r_{2}}
$$

$(\hat{\varphi} \otimes \widehat{\varphi})\left(\hat{\Gamma}\left(\lambda_{p q}^{r}\right)\left(\lambda_{p^{\prime} q^{\prime}}^{r^{\prime}} \otimes \lambda_{p^{\prime \prime} q^{\prime \prime}}^{r^{\prime \prime}}\right)\right)$

$$
\begin{aligned}
& =n(\operatorname{tr} \otimes \operatorname{tr})\left(\sum_{\substack{p_{1}, q_{1}, r_{1} \\
p_{2}, q_{2}, r_{2}}} C_{p_{1} q_{1} r_{1} p_{2} q_{2} r_{2}}^{p q r} \lambda_{p_{1}}^{r_{1}} \lambda_{p^{\prime} q^{\prime}}^{r^{\prime}} \otimes \lambda_{p_{2} q_{2}}^{r_{2}} \lambda_{p^{\prime \prime} q^{\prime \prime}}^{r^{\prime \prime}}\right) \\
& =n(\operatorname{tr} \otimes \operatorname{tr})\left(\sum_{p_{1}, p_{2}} C_{p_{1} p^{\prime} r^{\prime} p_{2} p^{\prime \prime} r^{\prime \prime}}^{p q r} \lambda_{p_{1} q^{\prime}}^{r^{\prime}} \otimes \lambda_{p_{2} q^{\prime \prime}}^{r^{\prime \prime}}\right) \\
& =\frac{n_{r^{\prime}} n_{r^{\prime \prime}}}{n} C_{q^{\prime} p^{\prime} r^{\prime} q^{\prime \prime} p^{\prime \prime} r^{\prime \prime}}^{p r} .
\end{aligned}
$$

By (3.9), we have the following identity. 


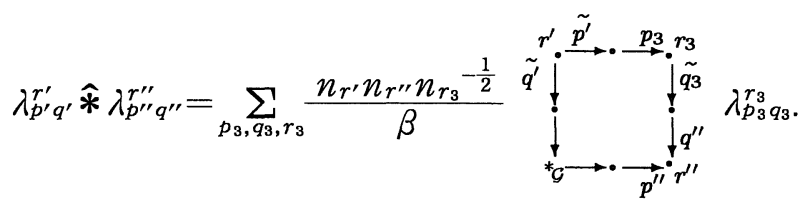

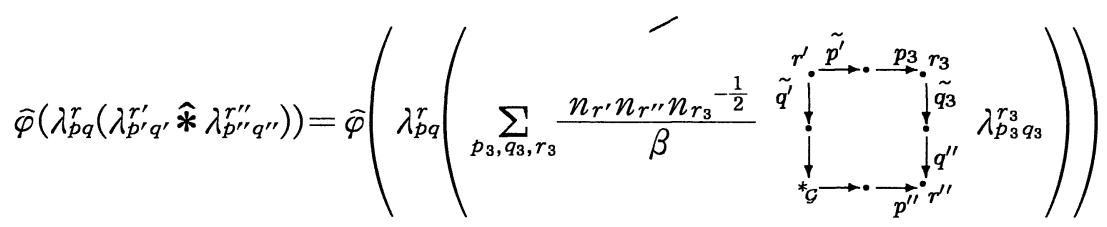

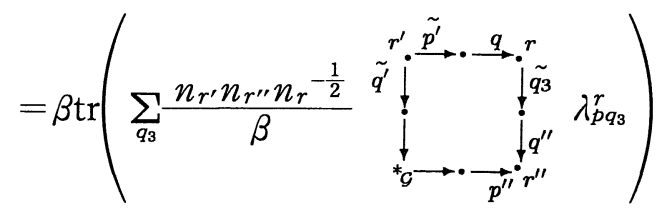

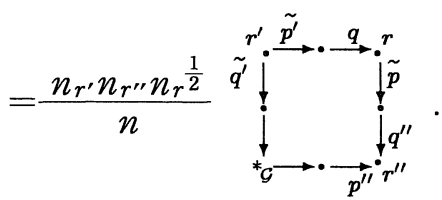

So we get the following equation.

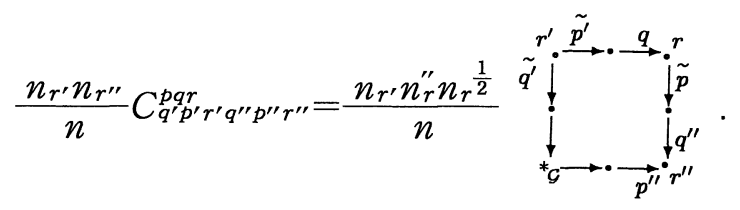

That is,

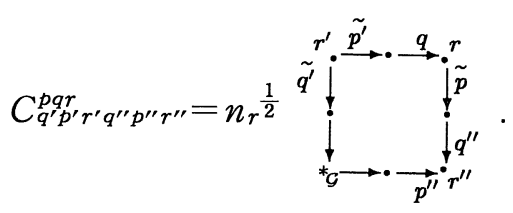

Thus we get (4.5).

Q. E. D.

Definition 4.4. We define the linear maps $W$ and $\widehat{W}$ by the following equations.

$$
\begin{aligned}
& \widehat{W}(x \otimes y):=\hat{\Gamma}(y)(x \otimes 1), x, y \in A_{0,2} . \\
& W(x \otimes y):=\Gamma(y)(x \otimes 1), x, y \in A_{1,1} .
\end{aligned}
$$

Lemma 4.5. The linear maps $\widehat{W}$ and $W$ are described explicitly as 
follows.

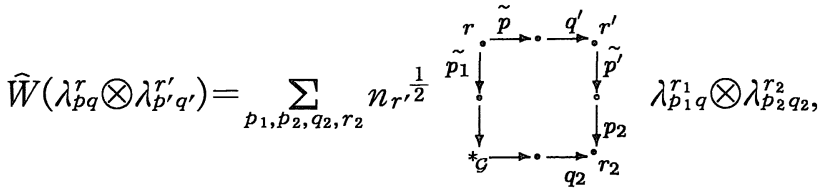

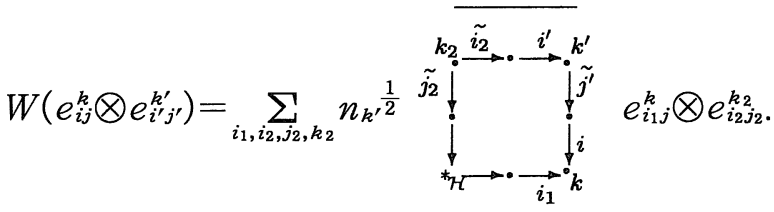

Proof. We can check these formulae by direct computations using Proposition 4.3 and Definition 4.4.

Q. E. D.

Lemma 4.6. The linear maps $\widehat{W}$ and $W$ are unitary operators.

Proof. First we shall derive the formula for $\widehat{W}^{*}$.

$$
\left(\widehat{W}\left(\lambda_{p q}^{r} \otimes \lambda_{p^{\prime} q^{\prime}}^{r^{\prime}}\right), \lambda_{p^{\prime \prime} q^{\prime \prime}}^{r^{\prime \prime}} \otimes \lambda_{p^{\prime \prime \prime} q^{\prime \prime \prime}}^{r^{\prime \prime \prime}}\right)=\left(\lambda_{p q}^{r} \otimes \lambda_{p^{\prime} q^{\prime}}^{r^{\prime}}, \bar{W}^{*}\left(\lambda_{p^{\prime \prime} q^{\prime \prime}}^{r^{\prime \prime}} \otimes \lambda_{p^{\prime \prime \prime} q^{\prime \prime \prime}}^{r^{\prime \prime \prime}}\right)\right) .
$$

Set

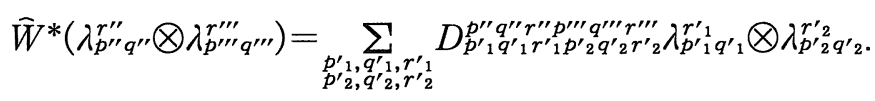

Computing the left hand side, we get the following.

$\left(\widehat{W}\left(\lambda_{p q}^{r} \otimes \lambda_{p^{\prime} q^{\prime}}^{r^{\prime}}\right), \lambda_{p^{\prime \prime} q^{\prime \prime}}^{r^{\prime \prime}} \otimes \lambda_{p^{\prime \prime \prime} q^{\prime \prime \prime}}^{r^{\prime \prime \prime}}\right)$

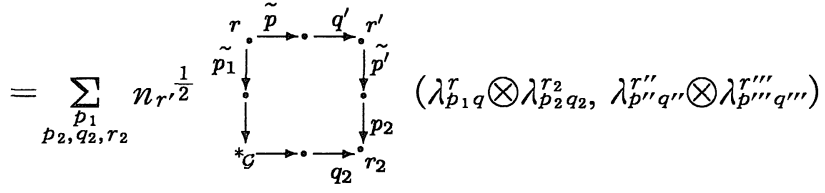

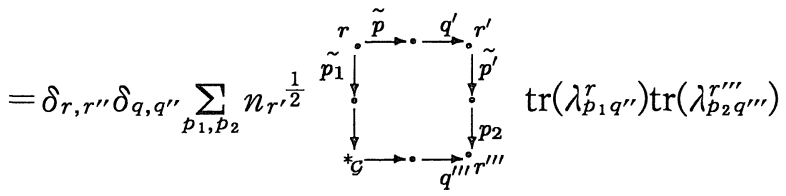

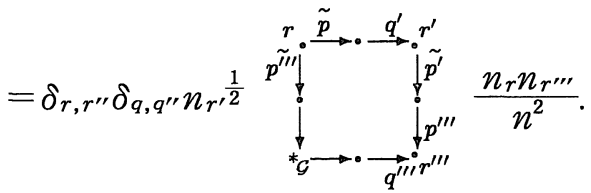

Computing the right hand side, we get the following. 
$\left(\lambda_{p q}^{r} \otimes \lambda_{p^{\prime} q^{\prime}}^{r^{\prime}}, \widehat{W}^{*}\left(\lambda_{p^{\prime \prime} q^{\prime \prime}}^{\gamma^{\prime \prime}} \otimes \lambda_{\left.p^{\prime \prime \prime \prime} q^{\prime \prime \prime}\right)}^{\gamma^{\prime \prime \prime}}\right)\right)$

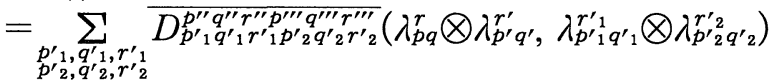

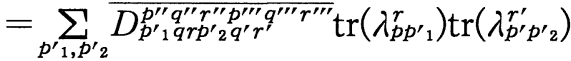

$$
\begin{aligned}
& =\frac{n_{r} n_{r^{\prime}}}{n^{2}} \overline{D_{p q r p^{\prime} q^{\prime} r^{\prime \prime}}^{p^{\prime \prime} q^{\prime \prime}} r^{\prime \prime \prime} q^{\prime \prime \prime} r^{\prime \prime \prime}} \text {. }
\end{aligned}
$$

Thus we get the following.

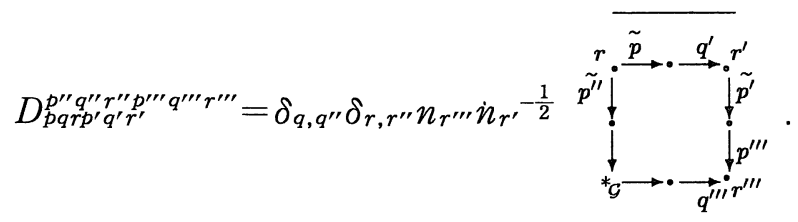

That is, we get the following formula for $\widehat{W}^{*}$.

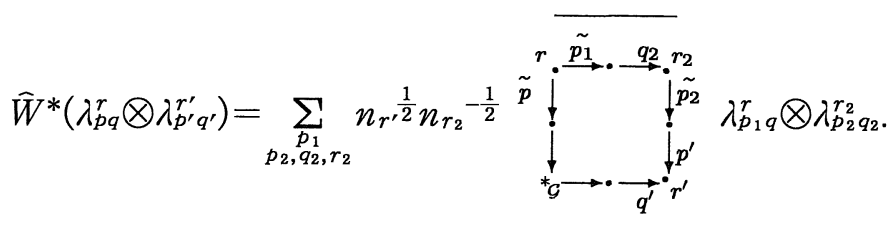

We can verify that $\widehat{W}^{*}$ is a unitary operator as follows.

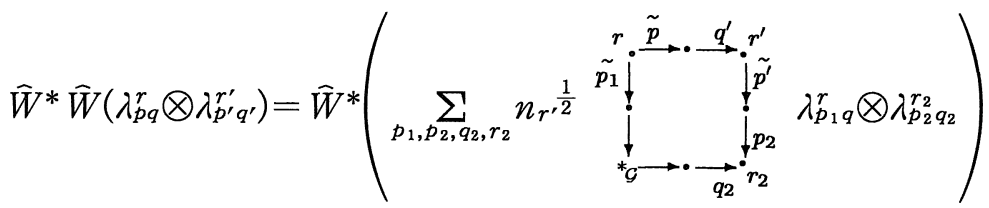

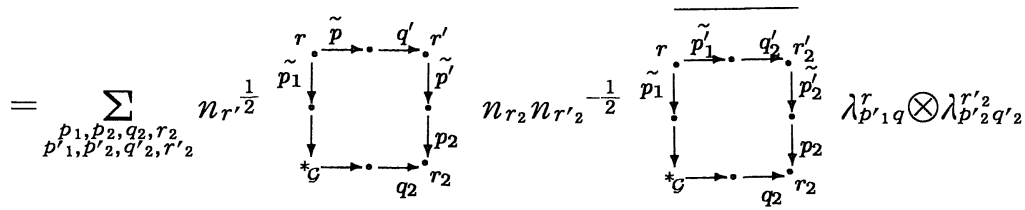

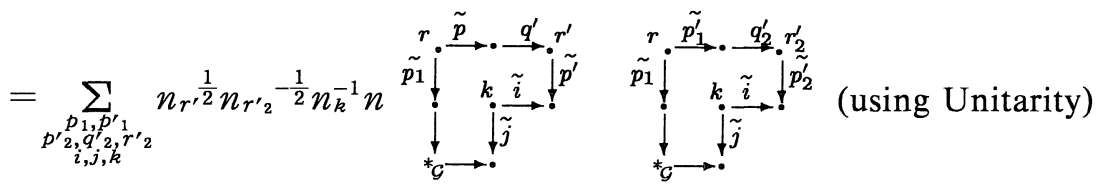

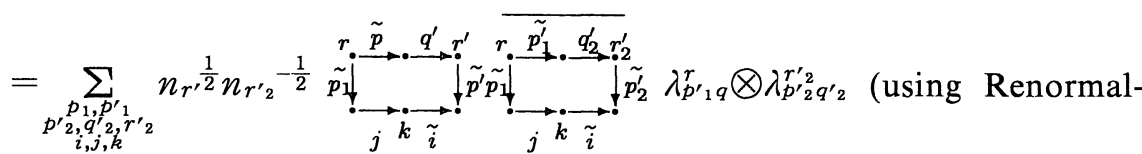

ization rule) 


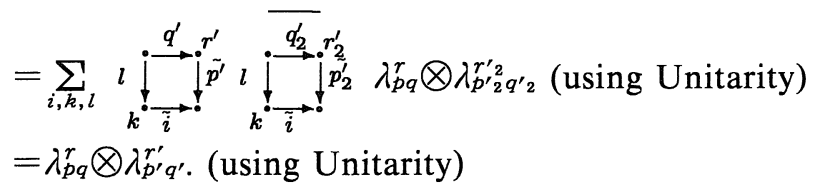

Thus we get $\hat{W}^{*} \hat{W}=i d_{A_{0,2} \otimes A_{0,2}}$. Since the linear map $\widehat{W}$ is on a finite dimensional Hilbert space $A_{0,2} \otimes A_{0,2}$, we get $\widehat{W} \widehat{W}^{*}=i d_{A_{0,2} \otimes A_{0,2}}$ automatically.

This completes the proof that $\widehat{W}$ is a unitary operator.

Similarly, we get the following formula for $W^{*}$.

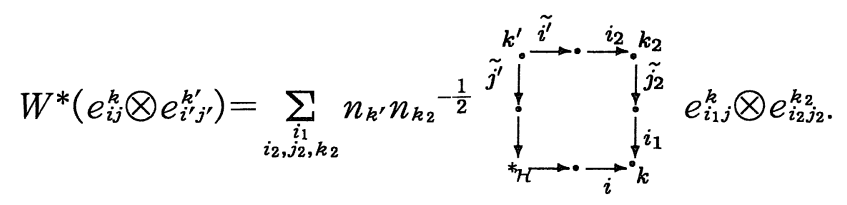

Using (4.12), we can prove that the linear map $W$ is a unitary operator on $A_{1,1} \otimes A_{1,1}$ in a similar way. So we omit the proof for $W$.

Q. E. D.

Definition 4.7 ([B-S $]$ ). Let $\mathscr{H}$ be a Hilbert space.

A unitary operator $V$ on $\mathscr{H} \otimes \mathscr{H}$ is called a multiplicative unitary if $V$ satisfies the following identity.

$$
V_{23} V_{12}=V_{12} V_{13} V_{23}
$$

Theorem 4.8. The following conditions for a bi-unitary connection are equivalent.

(i) The bi-unitary connection is flat for $*_{y}$ (resp. $*_{\mathscr{H}}$ ).

(ii) The unitary operator $\hat{W}^{*}$ (resp. $W^{*}$ ) defined in Definition 4.4 is a multiplicative unitary.

Proof. Applying * to (4.13) for $V=\widehat{W}^{*}$ we get the following equation.

$$
\widehat{W}_{12} \widehat{W}_{23}=\widehat{W}_{23} \widehat{W}_{13} \widehat{W}_{12} \text {. }
$$

So we will check that $\widehat{W}$ satisfies (4.14).

$\widehat{W}_{12} \widehat{W}_{23}\left(\lambda_{p q}^{r} \otimes \lambda_{p^{\prime} q^{\prime}}^{r^{\prime}} \otimes \lambda_{p^{\prime \prime} q^{\prime \prime}}^{r^{\prime \prime}}\right)$

$=\widehat{W}_{12}\left(\lambda_{p q}^{r} \otimes \widehat{W}\left(\lambda_{p^{\prime} q^{\prime}}^{r^{\prime}} \otimes \lambda_{p^{\prime \prime} q^{\prime \prime}}^{r^{\prime \prime}}\right)\right)$

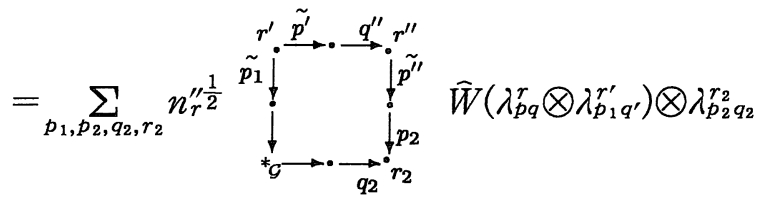




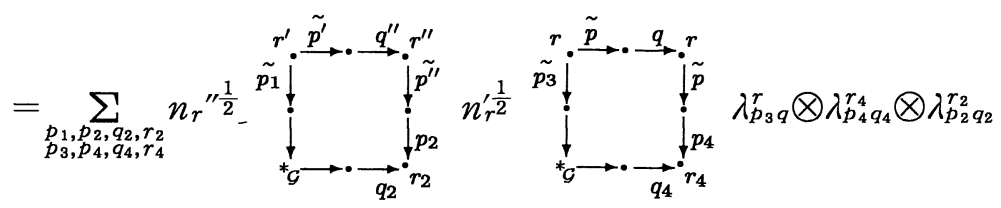

$\widehat{W}_{23} \widehat{W}_{13} \widehat{W}_{12}\left(\lambda_{p q}^{r} \otimes \lambda_{p^{\prime} q^{\prime}}^{r^{\prime}} \otimes \lambda_{p^{\prime \prime} q^{\prime \prime}}^{r^{\prime \prime}}\right)$

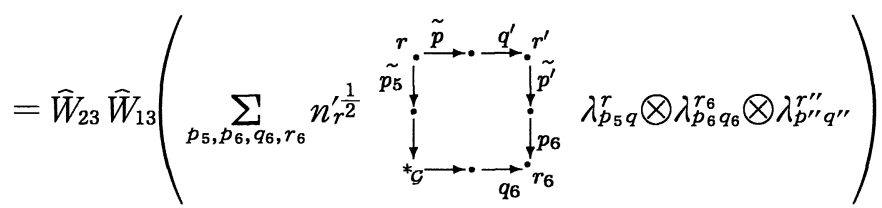

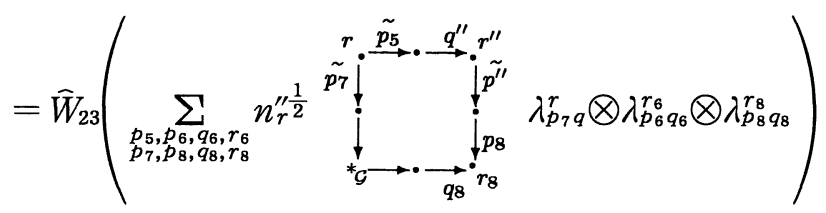

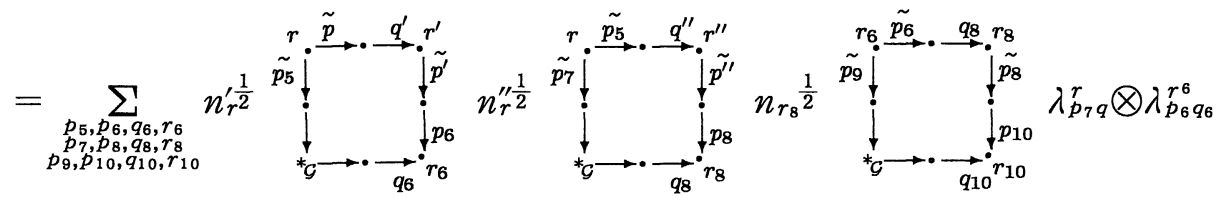

$\otimes \lambda_{p_{10} q_{10}}^{r_{10}}$

So to check that $\widehat{W}$ satisfies (4.14), we must prove the following identity.

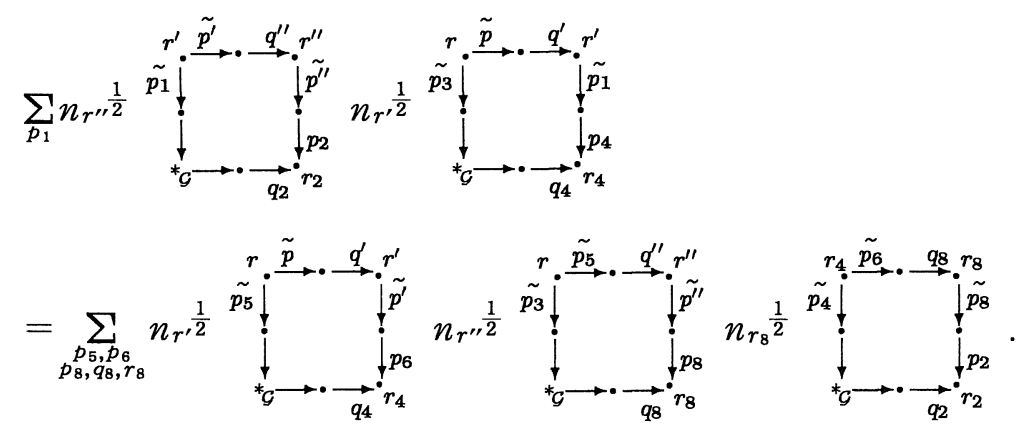

We multiply formula (4.15) by $n_{r^{\prime \prime}}$ and $r^{\prime \prime}$. Then using Unitarity, we get the following identity equivalent to (4.15). 

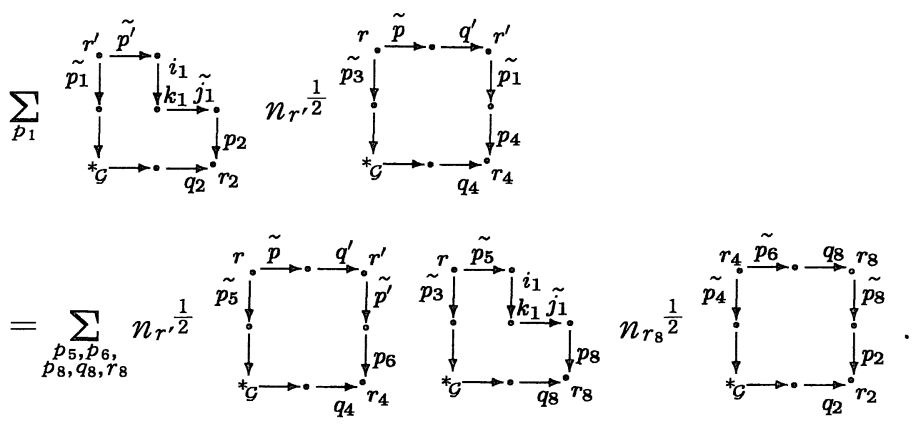

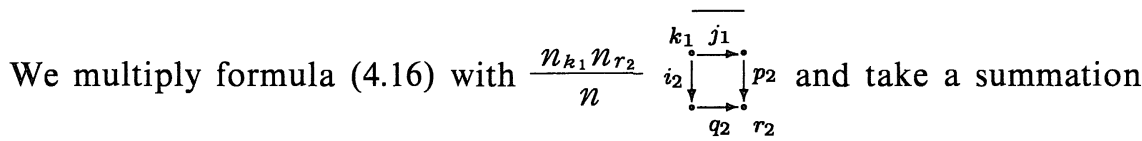
over $p_{2}, q_{2}$ and $r_{2}$. Then using Unitarity to the both hand sides and Renormalization rule to the left hand side, we get the following identity equivalent to (4.16).
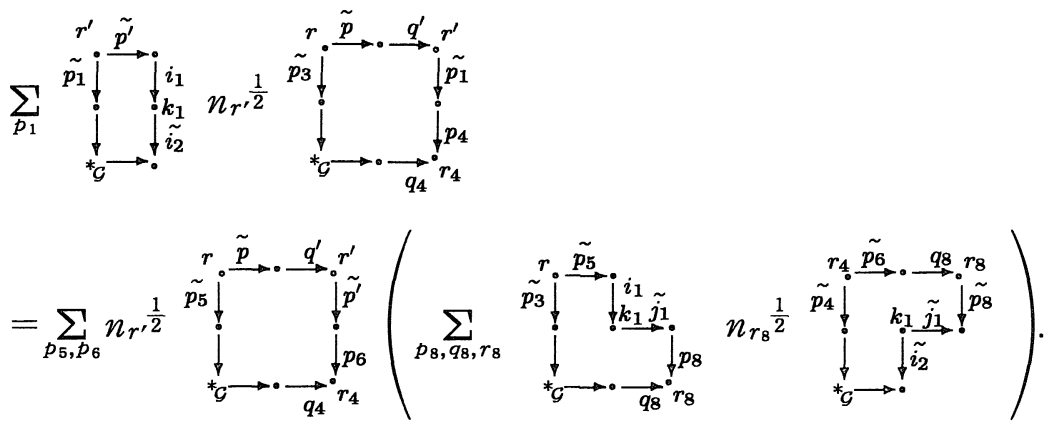

Using Renormalization rule to the last term of the right hand side, we get the following identity equivalent to (4.17).
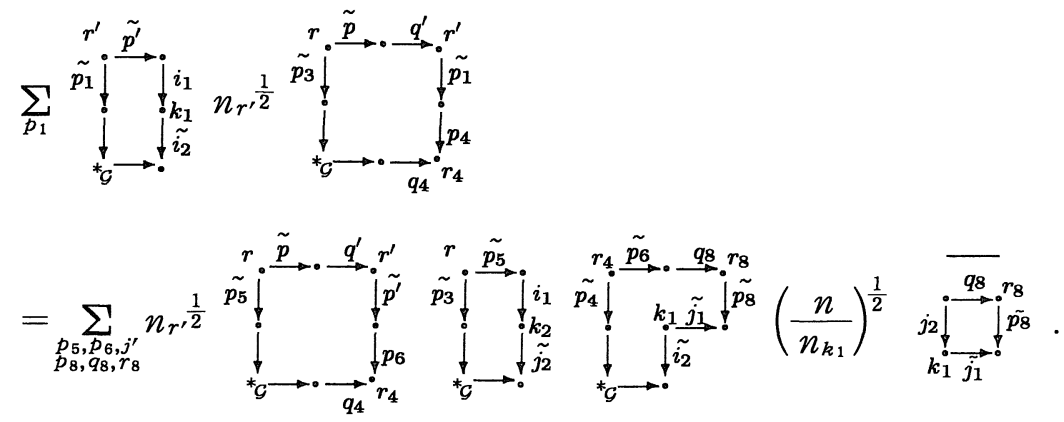

Using Unitarity to $p_{8}, q_{8}$ and $r_{8}$, we get the following identity equivalent to (4.18). 
(4.19)

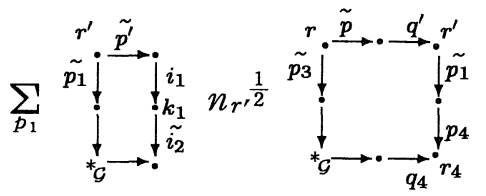

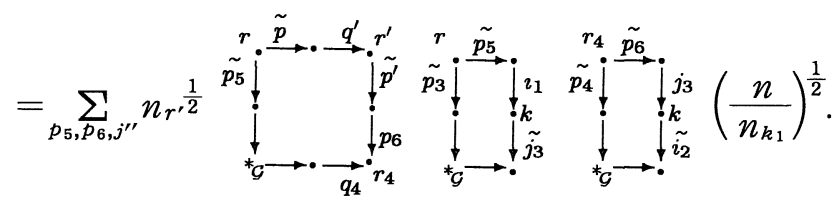

Using Renormalization rule to the both hand sides of (4.19), we get the following identity equivalent to (4.19).

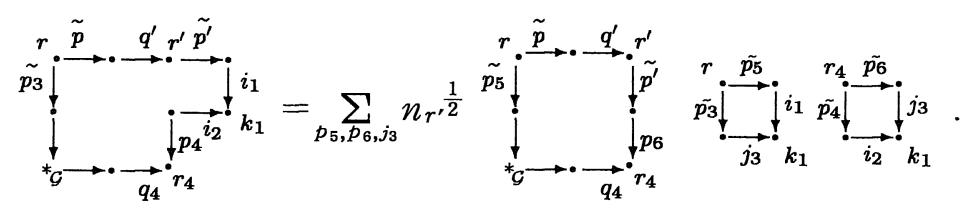

We multiply formula (4.20) by $i_{2}$. Then using Unitarity, we get the following identity equivalent to (4.20).

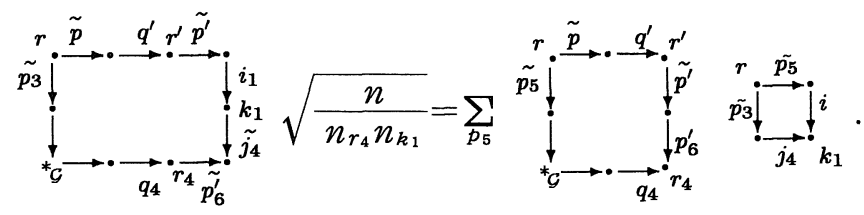

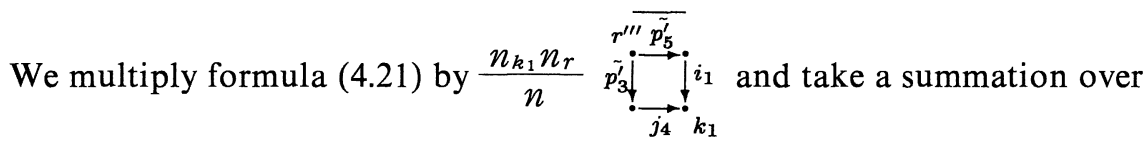
$i_{1}, k_{1}$ and $j_{4}$. Then using Unitarity, we get the following identity equivalent to (4.21).

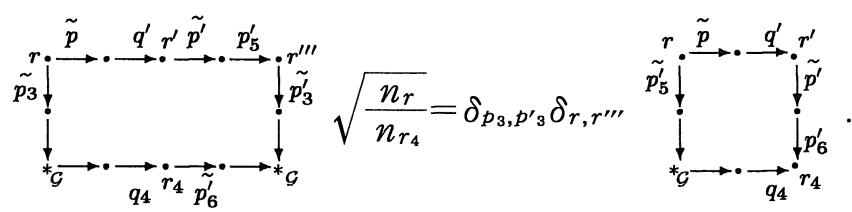
$\widehat{W}^{*}$.

Thus we get the following identity equivalent to the pentagonal relation for 


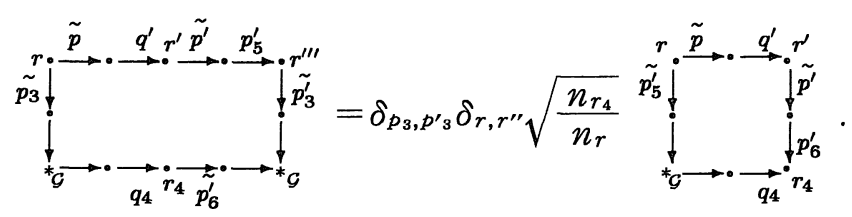

Using condition 2 in Definition 1.4, the identity (4.23) implies the flatness condition for $*_{g}$.

We can also prove that the pentagonal relation for $W^{*}$ implies the flatness condition for $*_{F b}$ in a similar way. So we omit the proof for $W^{*}$. Thus condition (ii) implies condition (i).

Next, we show that condition (i) implies (ii).

We multiply formula (4.21) by $\frac{n_{k} n_{r}}{n} \stackrel{r}{\overrightarrow{p_{3}}} \stackrel{\overrightarrow{p_{5}^{\prime}}}{\rightarrow} \vec{j}_{j_{4}}^{i} i_{1}$ and take a summation over $i_{1}, k_{1}$ and $j_{4}$. Then using Unitarity, we get the following identity equivalent to (4.21).

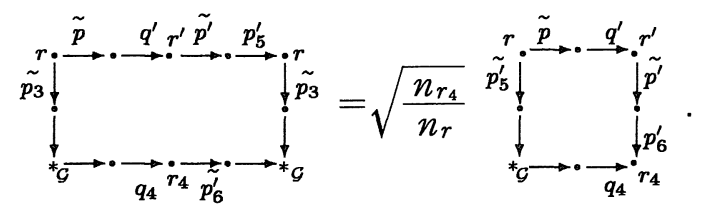

We multiply formula (4.24) by and $r^{\prime}$. Then using Unitarity and Renormalization rule, we get the following identity equivalent to (4.24).

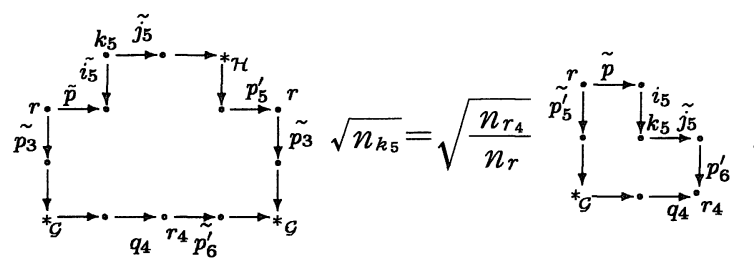

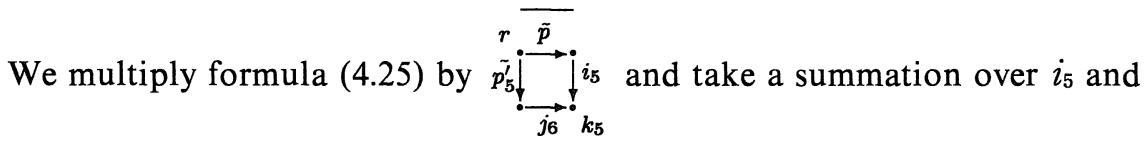
p. Then using Unitarity we get the following identity equivalent to (4.25). 


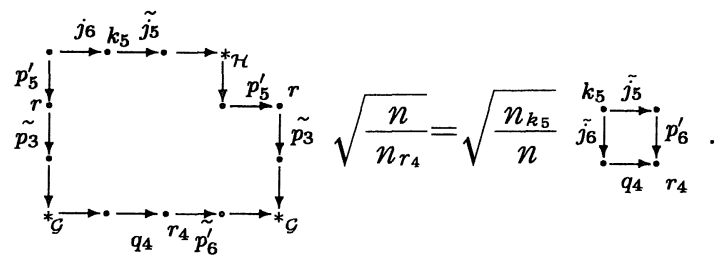

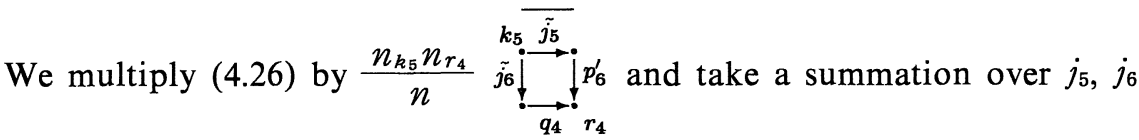
and $k_{5}$. Then using Unitarity, we get the following identity equivalent to (4.26).

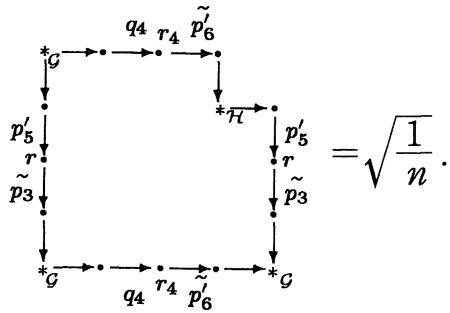

By flatness, we get the following identity.

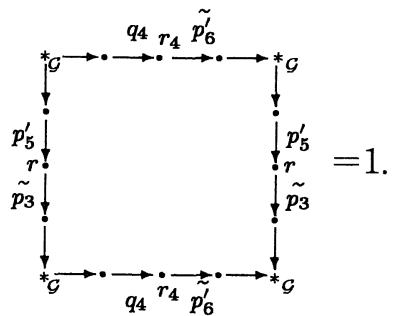

We can decompose (4.28) as follows.

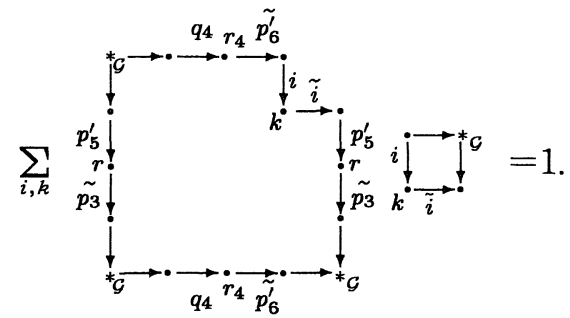

We can get 


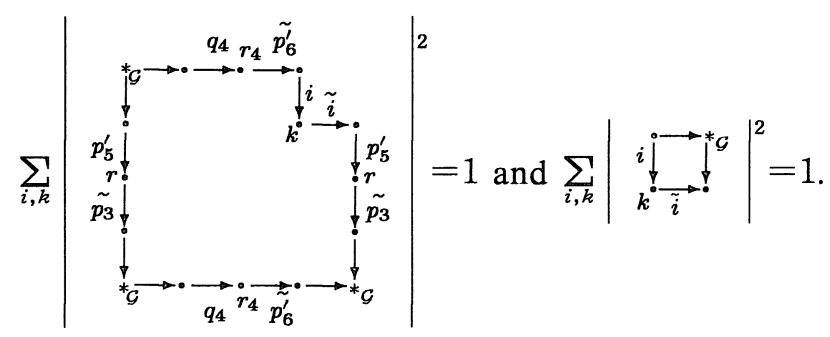

because of Unitarity.

By the Cauchy-Schwarz inequality, we get

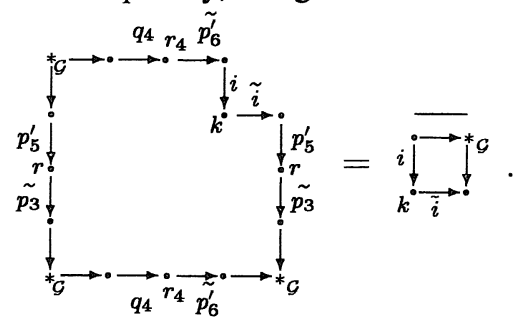

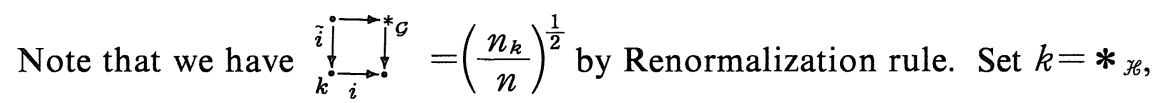
then we get identity (4.27). Thus we are done.

Q. E. D.

We apply the construction of Hopf $\mathrm{C}^{*}$-algebras by Baaj-Skandalis to the above multiplicative unitaries. Since we know that a finite dimensional Hopf $\mathrm{C}^{*}$-algebra is a finite dimensional $\mathrm{Kac}$ algebra and vice versa, we get the following corollary.

Corollary 4.9. In the case of a paragroup with depth two, we have Kac algebra structures in $A_{1.1}, A_{0.2}$.

\section{§5. Duality between Kac Algebra $A_{0,2}$ and Kac Algebra $A_{1,1}$}

In the previous section, we have constructed Kac algebra structures on $A_{1,1}$ and $A_{0,2}$. Those algebras have two algebra structures, string algebras and convolution algebras. The products of $C^{*}$-algebra structures in $A_{1,1}$ and $A_{0,2}$ are closely related. The two products in $A_{0,2}$ are given by exchanging the two products in $A_{1,1}$. So the corresponding Kac algebras have a very simple relation, which is called duality in Kac algebra theory. For applications, we will describe this duality between $A_{0,2}$ and $A_{1,1}$ by comparing the formulae of the fundamental unitaries $W$ and $\widehat{W}$. For our purpose, we shall compute formulae of $W$ and $\hat{W}$. We need some preparations. 
In this section, we fix a paragroup with depth 2 .

Lemma 5.1. We have the following formulae.

$$
\begin{aligned}
& (x * y)^{*}=x^{*} * y^{*}, x, y \in A_{1,1}, \\
& (x \hat{*} y)^{*}=x^{*} \hat{*} y^{*}, x, y \in A_{0,2} .
\end{aligned}
$$

Proof. As we can prove the above two formulae in a similar way, we will prove only (5.2).

It is enough to prove (5.2) for the basis in $A_{0,2}$.

By (3.9), we get the following.

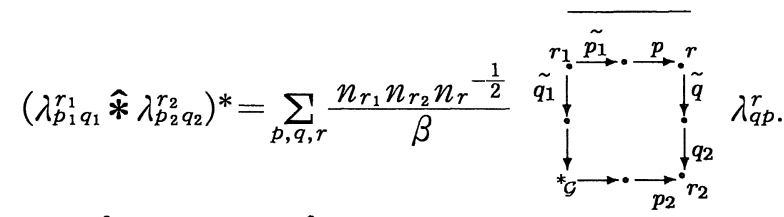

$$
\begin{aligned}
& \lambda_{p_{1} q_{1}}^{r_{1} *} \hat{*} \lambda_{p_{2} q_{2}}^{r_{2} *}=\lambda_{q_{1} p_{1}}^{r_{1}} \hat{*} \lambda_{q_{2} p_{2}}^{r_{2}}
\end{aligned}
$$

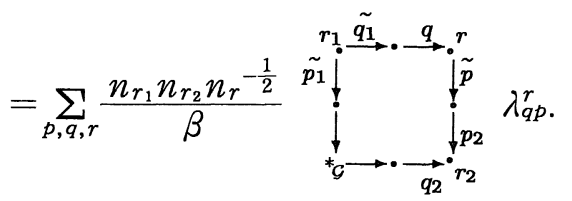

Thus we have shown the following identity.

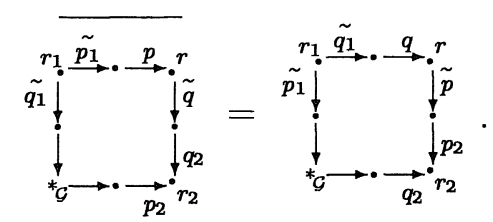

Applying (4.23) to the left hand side and right hand side of (5.3), we get the following.

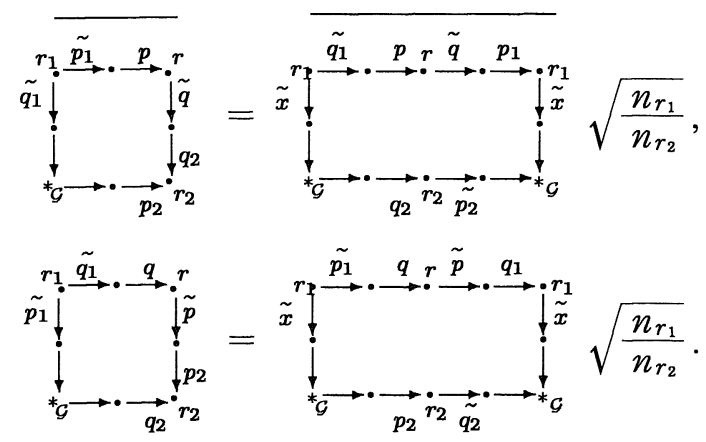

By Renormalization rule axiom, the above two values are equal. Thus we 
are done.

Q. E. D.

We list the identities we had above as a lemma.

Lemma 5.2. We have the following identities.
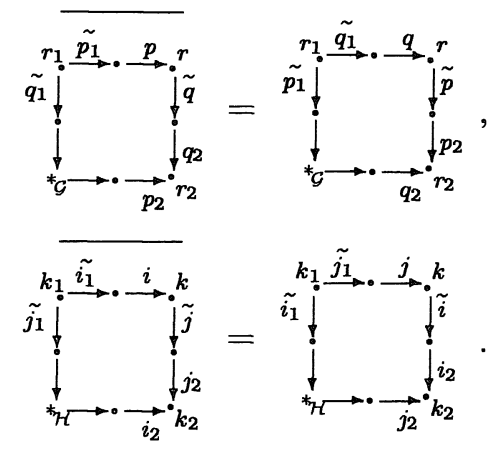

To compare the structures of the two Kac algebras $A_{0,2}$ and $A_{1,1}$, we transform the string algebra structure and the convolution algebra structure in $A_{0,2}$ to $A_{1,1}$ by the Fourier transform. Since we know the Fourier transform gives an isomorphism from the string algebra $A_{0,2}$ onto the convolution algebra $A_{1,1}$ by formulae (3.7), (3.8), (3.13) and (3.14) and the unitarity of Fourier transform, we can recover the Kac algebra structure of $A_{0,2}$ in $A_{1,1}$. We use the same notations in the case of the Kac algebra constructed on $A_{0,2}$.

We can define $\widehat{\Gamma}$ and $\widehat{W}$ as before and get the following formula.

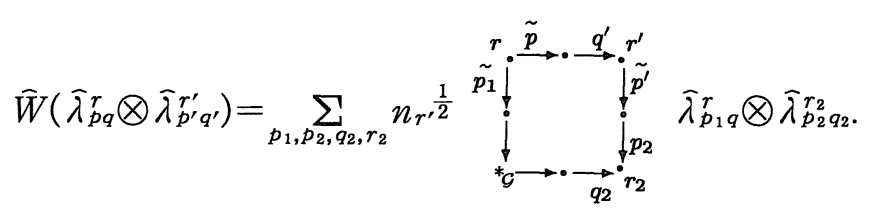

We shall derive another formula.

We multiply (5.6) by $n_{r^{2}}^{-\frac{1}{2}}$

$r^{\prime}$. Using Unitarity, we get the following formula equivalent to (5.6).

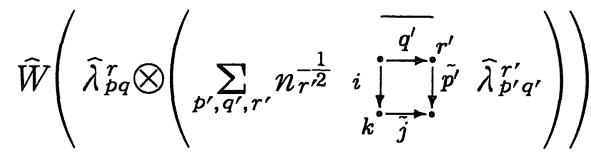

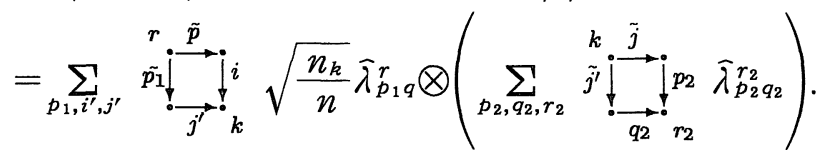


In the above equality, we used Renormalization rule to the term including the vertex $*_{g}$.

We compute a term in the left hand side of (5.7).

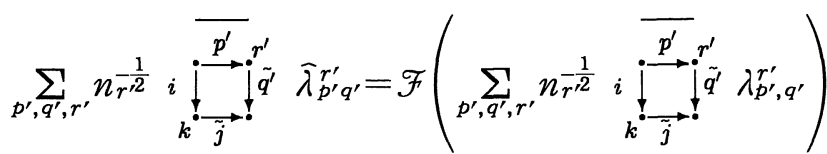

$$
\begin{aligned}
& =n_{k}{ }^{-\frac{1}{2}} \mathscr{F}\left(\tilde{F}\left(e_{i j}^{k}\right)^{*}\right) \\
& =n_{k}{ }^{-\frac{1}{2}} e_{i j}^{k \#} \text {. }
\end{aligned}
$$

Similarly, we can compute the right hand side of (5.7) as follows.

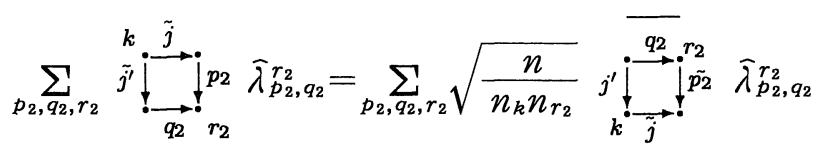

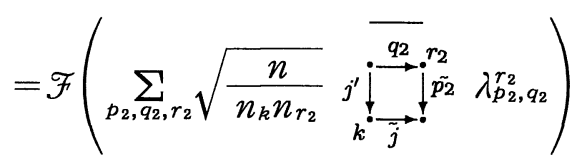

$$
\begin{aligned}
& =\frac{\sqrt{n}}{n_{k}} \mathscr{F}\left(\tilde{F}\left(e_{j^{\prime} j}^{k}\right)^{*}\right) \\
& =\frac{\sqrt{n}}{n_{k}} e_{j^{\prime} j}^{k \# .}
\end{aligned}
$$

In the first equality, we have used Renormalization rule.

Thus we get the following formula equivalent to (5.6).

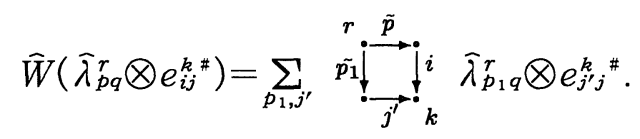

Using Lemma 5.1, we get the following formula for $\widehat{W}$.

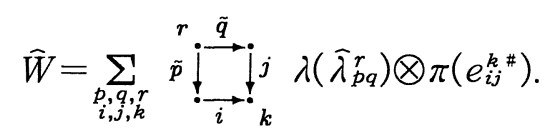

Next, we shall derive another formula from (4.12) for $W^{*}$.

We multiply (4.12) by $n_{k^{\prime}}-\frac{1}{2}$ $k^{\prime}$. Using Unitarity axiom, we get the following formula equivalent to (4.12).

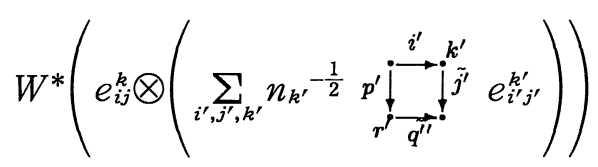




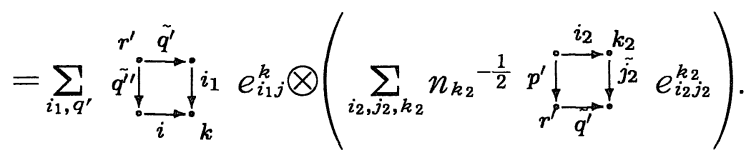

We can compute a term in the left hand side as follows.

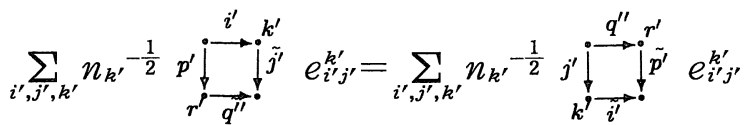

$$
\begin{aligned}
& =n_{r^{\prime}}-\frac{1}{2} \mathscr{F}\left(\lambda_{q^{\prime \prime} p^{\prime}}^{r^{\prime}}\right)^{*} \\
& =n_{r^{\prime}}{ }^{-\frac{1}{2}} \hat{\lambda}_{q^{\prime \prime} p^{\prime}}^{r^{\prime}} \text {. }
\end{aligned}
$$

Similarly, we can deform the right hand side and get the following formula equivalent to (4.12).

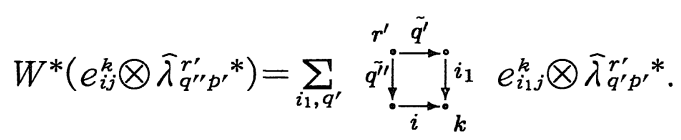

Using Lemma 5.1, we get the following formula for $W^{*}$.

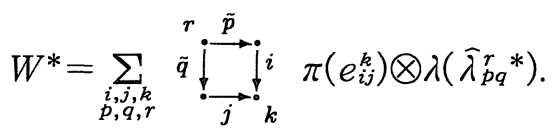

We have arrived at the main point in this section.

Proposition 5.3. We have the following relation between the fundamental unitaries $W$ and $\widehat{W}$.

$$
\widehat{W}=\Sigma W^{*} \Sigma \text {. }
$$

Here $\Sigma$ means the flip map on $M \otimes M$.

So the Kac algebra constructed on $A_{0,2}$ and that on $A_{1,1}$ are dual to each other.

Proof. We use formulas (5.8) and (5.9). In formula (5.8), $e_{i j}^{k \#}$ is expressed with $e_{i j}^{k \prime} s$ as follows.

$$
\begin{aligned}
& e_{i j}^{k \#}=\mathscr{F}\left(\hat{F}\left(e_{i j}^{k}\right)^{*}\right) \\
& =\mathscr{F}\left(\sum_{p, q, r}\left(\frac{n_{k}}{n_{r}}\right)^{\frac{1}{2}}{ }_{i} \underset{{ }_{k}}{\stackrel{p}{\longrightarrow} \vec{j}_{j}^{r} \tilde{q}} \lambda_{q p}^{r}\right)
\end{aligned}
$$




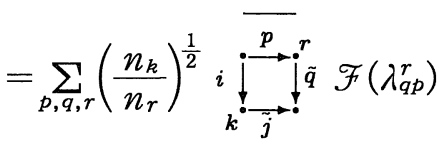

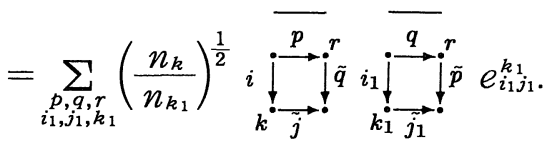

Similarly, in formula (5.8), $\hat{\lambda}_{p q}^{r}{ }^{*}$ is expressed with $\hat{\lambda}_{p q}^{r}$ 's as follows.

$$
\begin{aligned}
& \bar{\lambda}_{p q}^{r}{ }^{*}=\mathcal{F}\left(\lambda_{p q}^{r}\right)^{*} \\
& =\sum_{i, j, k}\left(\frac{n_{r}}{n_{k}}\right)^{\frac{1}{2}} i_{k} \stackrel{p}{\longrightarrow} \stackrel{j}{j}^{r} \tilde{q} e^{k} e_{j i}^{k}
\end{aligned}
$$

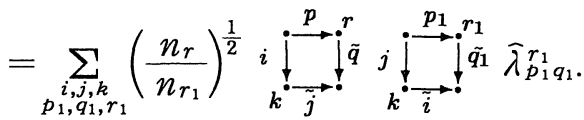

Using these formulas, we can write $\widehat{W}$ and $W^{*}$ as follows.

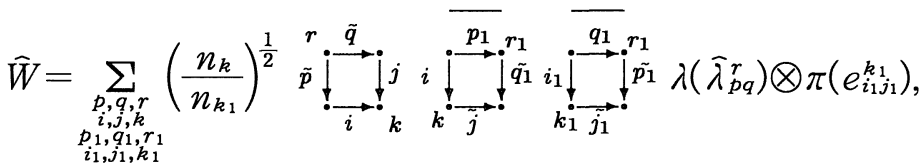

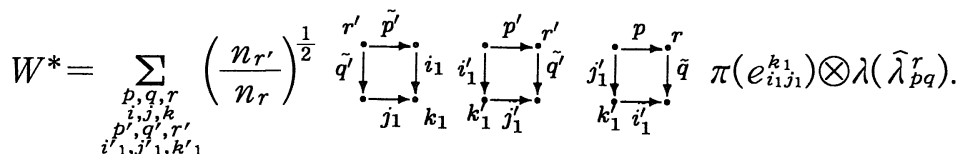

So we must show the following identity.

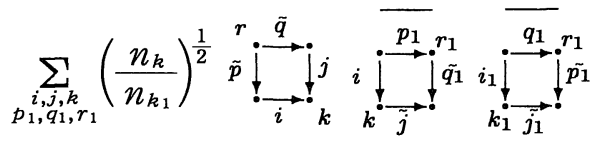

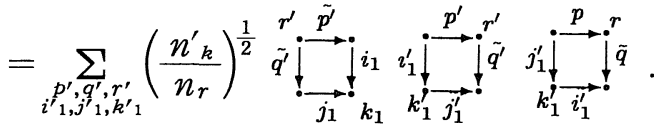

Applying Renormalization rule to the first term of the right hand side of (5.10), we get the following identity equivalent to (5.10).

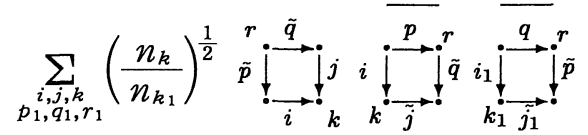

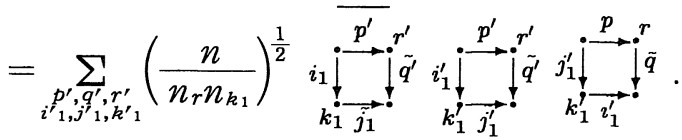


We multiply (5.11) by Using Unitarity, we get the following identity equivalent to (5.11).

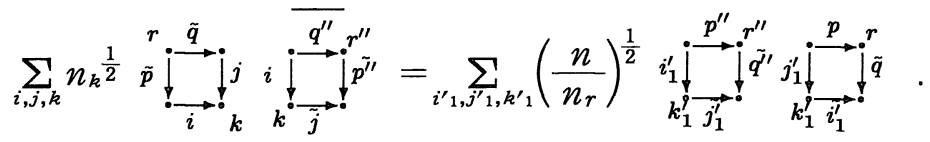

Using Renormalization rule to the first term of the left hand side of (5.12), we get the following identity equivalent to (5.12).

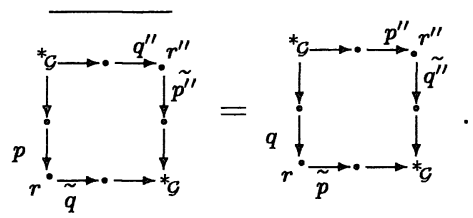

So we must show this identity.

By Lemma 5.2, set $r=*_{\rho}$ and apply Renormalization rule to the both hand sides, then we get (5.12). Thus we are done.

Q. E. D.

\section{§6. A Realization of a Paragroup with Depth Two}

We realize a paragroup with depth two from an initial Kac algebra and describe the subfactor arising from the paragroup.

Suppose $\mathbf{K}=(\mathcal{M}, \Gamma, \varkappa, \varphi)$ is a finite dimensional $\mathrm{Kac}$ algebra. We denote the left regular representation for this Kac algebra by $\pi$ and identify the original Kac algebra and the represented algebra. Because this algebra is a finite dimensional $C^{*}$-algebra, we may assume that $\mathcal{M}$ and the Haar measure $\varphi$ are of the following form.

$$
M=\bigoplus_{k=1}^{l} M_{n_{k}}(\mathbf{C}), \varphi=\frac{1}{n} \sum_{k=1}^{l} n_{k} \operatorname{Tr}_{M_{n_{k}}(\mathbf{C})}
$$

Here $n=\operatorname{dim} \mathcal{M}$.

We can construct the dual Kac algebra $\hat{\mathbf{K}}=(\hat{M}, \hat{\Gamma}, \hat{x}, \widehat{\varphi})$ from the initial $\mathrm{Kac}$ algebra $\mathrm{K}([\mathrm{E}-\mathrm{S}])$. We denote the left regular representation by $\lambda$ and identify the original dual Kac algebra and the represented one. As above, we may assume that $\widehat{M}$ and the Haar measure $\hat{\varphi}$ are of the following form.

$$
\widehat{M}=\bigoplus_{r=1}^{s} M_{n_{r}}(\mathbf{C}), \hat{\varphi}=\sum_{r=1}^{s} n_{k} \operatorname{Tr}_{M_{n_{r}}(\mathbf{C})} \text {. }
$$

Note that we have the Plancherel formula for the inner products arising from the Haar measures $([\mathrm{K}-\mathrm{P}])$. That is, 


$$
\varphi\left(a b^{*}\right)=\hat{\varphi}\left(a * b^{\#}\right) .
$$

We shall construct a string algebra from these data. At first, we set the two graphs, one from the Kac algebra and the other from the dual Kac algebra. More precisely, the graph $\mathscr{G}$ (resp. $\mathscr{H}$ ) has the unique vertex that is connected to the vertices corresponding to the direct summands of the multi-matrix algebra $M$ (resp. $\widehat{M}$ ) by $n_{k}$ (resp. $n_{r}$ ) edges. We fix a vertex corresponding to the one dimensional representation for $\pi$ (resp. $\lambda$ ) as a special vertex $*_{y}$ (resp. $*_{\mathscr{H}}$ ).

Next, we introduce a bi-unitary connection on above two graphs. Connect these graphs as in the first figure in section 2.

Lemma 6.1. We have the following formulae.

$$
\begin{aligned}
& \lambda_{p q}^{r}=\sum_{i, j, k} \frac{n}{n_{k}} \varphi\left(e_{j i}^{k} \lambda_{p q}^{r}\right) e_{i j}^{k}, \\
& e_{i j}^{k}=\sum_{p, q, r} \frac{1}{n_{r}} \overline{\varphi\left(\lambda_{p q}^{r} e_{j i}^{k}\right)} \lambda_{p q}^{r} .
\end{aligned}
$$

Proof. Since we can easily deduce these formulae by using (6.1), we omit the proof.

Q. E. D.

Proposition 6.2. We can define a bi-unitary connection on the above two graphs by the following formula.

$$
\tilde{j} \underset{\varliminf_{q}}{\stackrel{\tilde{i}}{\longrightarrow} !} p=\frac{n}{n_{k} n_{r}} \varphi\left(e_{i j}^{k} \lambda_{p q}^{r}\right) .
$$

Here $e_{i j}^{k}$ (resp. $\left.\lambda_{p q}^{r}\right)$ means the system of matrix units corresponding to the decomposition of $\mathcal{M}$ (resp. $\widehat{M})$.

Proof. As described in [S], we have the following identity for the fundamental unitary $W$ for the Kac algebra $\mathbf{K}$.

$$
W=\sum_{\substack{i, j, k \\ p, q, r}} \frac{n}{n_{k} n_{r}} \varphi\left(e_{i j}^{k} \lambda_{p q}^{r}\right) \pi\left(e_{i j}^{k}\right) \otimes \lambda\left(\lambda_{p q}^{r}\right)
$$

Also we have

$$
W^{*}=\sum_{\substack{i, j, k \\ p, q, r}} \frac{n}{n_{k} n_{r}} \overline{\varphi\left(e_{i j}^{k} \lambda_{p q}^{r}\right)} \pi\left(e_{i j}^{k}\right) \otimes \lambda\left(\lambda_{p q}^{r}\right)
$$

Since the fundamental unitary is a unitary operator on a Hilbert space $M \otimes M$, we get the following identities.

$$
W W^{*}=i d_{\mu \otimes \mathcal{M}}, W^{*} W=i d_{M \otimes \mathcal{M}}
$$


It is easy to see that these identities give Unitarity for the connection defined above.

By Lemma 6.1, we have

$$
\begin{aligned}
\lambda_{p q}^{r} & =\sum_{i, j, k} \frac{n}{n_{k}} \varphi\left(e_{j i}^{k} \lambda_{p q}^{r}\right) e_{i j}^{k} \\
& =\sum_{p_{p^{\prime}, q_{j}, k, r^{\prime}}} \frac{n}{n_{k}} \varphi\left(e_{j i}^{k} \lambda_{p q}^{r}\right) \frac{1}{n^{\prime}{ }_{r}} \overline{\varphi\left(\lambda_{p^{\prime} q^{\prime}}^{r^{\prime}} e_{j i}^{k}\right)} \lambda_{p^{\prime} q^{\prime}}^{r^{\prime}} .
\end{aligned}
$$

Thus we get the following identity.

$$
\sum_{i, j, k} \frac{n}{n_{k} n_{k}^{\prime}} \varphi\left(e_{j i}^{k} \lambda_{p q}^{r}\right) \overline{\varphi\left(\lambda_{p^{\prime} q^{\prime}}^{r^{\prime}} e_{j i}^{k}\right)}=\delta_{p, p^{\prime}} \delta_{q, q^{\prime}} \delta_{r, r^{\prime}}
$$

We get the following identity in the same way.

$$
\sum_{i, j, k} \frac{n}{n^{\prime}{ }_{k} n_{r}} \varphi\left(e_{j^{\prime} i^{\prime}}^{k^{\prime}} \lambda_{p q}^{r}\right) \overline{\varphi\left(\lambda_{p q}^{r} e_{j i}^{k}\right)}=\delta_{i, i^{\prime}} \delta_{j, j^{\prime}} \delta_{k, k^{\prime}}
$$

It is easy to see that identities (6.5) and (6.6) give Renormalization rule. So we are done.

Q. E. D.

As we described in section 2 , we can construct the string algebra $A_{1,1}$ (resp. $\left.A_{0,2}\right)$ as $\mathcal{M}$ (resp. $\widehat{M}$ ). Thus we have Kac algebra structures in the string algebras $A_{1,1}$ and $A_{0,2}$. By Theorem 4.8, we can show that the above bi-unitary connection is a flat connection. So we have a paragroup for the two graphs $\mathscr{G}$ and $\mathscr{H}$. Thus we get a subfactor from this paragroup and denote it by $N \subset M$.

Sekine ( $[\mathrm{S}]$ ) computed the connection for a subfactor $P \subset P \times{ }_{\alpha} \mathbf{K}$ concretely. This connection is equal to the connection given in Proposition 6.2. Thus by Theorem 2.5 in section 2 , the subfactor $N \subset M$ constructed above and the subfactor $P \subset P \times{ }_{\alpha} \mathbf{K}$ are anti-isomorphic.

Theorem 6.3. Assume that we have a finite dimensional Kac algebra $\mathbf{K}$. We can construct a subfactor from the Kac algebra $\mathbf{K}$ and this subfactor is anti-isomorphic to the subfactor $P \subset P \times_{\alpha} \mathbf{K}$, where $P$ is an $A F D I I_{1}$ factor and $\alpha$ is an outer action of $\mathbf{K}$.

Remark. We can represent $N \subset M$ as a Kac algebra crossed product subfactor by describing an outer action of $\mathbf{K}$ on $M$ concretely ([Da]).

\section{Acknowledgements}

This paper is the author's master thesis at Department of Mathematical Sciences in University of Tokyo. He is much indebted to Prof. Kawahigashi for many discussions and expresses his gratitude. 


\section{References}

[B-G] de Boer, J. and Goeree, J., Markov traces and $\mathrm{II}_{1}$ factors in conformal field theory, Comm. Math. Phys., 139 (1991), 267-304.

[B-S] Baaj, S. and Skandalis, G., Unitaires multiplicatifs et dualité pour les produits croisés de C*-algèbres, Ann. Sci. Ec. Norm. Sup., 26 (1993), 425-488.

[Da] David, M.-C., Paragroupe d'Adrian Ocneanu et algèbre de Kac, Pac. J. Math., 172 (1996), 331-363.

[E] Enock, M., Produit croisé d'une algèbre de von Neumann par une algèbre de Kac, $J$. Funct. Anal., 26 (1977), 16-47.

[E-K1] Evans, D.E. and Kawahigashi, Y., From subfactors to 3-dimensional topological quantum field theories and back - a detailed account of Ocneanu's theory-, Internat. $J$. Math., 6 (1995), 537-558.

[E-K2] _ Subfactors and conformal field theory, in "Quantum and non-commutative analysis", 341-369, Kluwer Academic (1993).

[E-N] Enock, M. and Nest, R., Irreducible inclusions of factors, multiplicative unitaries, and Kac algebras, J. Funct. Anal., 137 (1996), 466-543.

[E-S] Enock, M. and Schwarz, M., Kac algebras and duality, Springer Verlag, 1992.

[G-H-J] Goodman, F., de la Harpe, P., and Jones, V.F.R., Coxeter graphs and towers of algebras, MSRI publications 14, Springer, 1989.

[H-O] Herman, R. and Ocneanu, A., Index theory and Galois theory for infinite inclusions of factors, C.R. Acad. Sci. Paris, 309 (1989), 923-927.

[Jo] Jones, V.F.R., Index for subfactors, Invent. Math., 72 (1983), 1-15.

[K] Kawahigashi, Y., On flatness of Ocneanu's connections on the Dynkin diagrams and classification of subfactors, J. Funct. Anal., 127 (1995), 63-107.

[K-P] Kac, G.I. and Paljutkin, V.G., Finite ring groups, Trans. Moscow Math. Soc., (1996), 251 $-294$.

[Lo] Longo, R., A duality for Hopf-algebras and for subfactors I, Comm. Math. Phys., 159 (1994), 133-150.

[M-S] Moore, G. and Seiberg, N., Classical and quantum conformal field theory, Comm. Math. Phys., 123 (1989), 177-254.

[O1] Ocneanu, A., Quantized group, string algebras and Galois theory for algebras, in Operator algebras and applications, Vol. 2 (Warwick, 1987), London Math. Soc. Lect. Note Series Vol. 136, Cambridge University Press, (1988), 119-172.

[O2] _ Graph geometry, quantized groups and nonamenable subfactors, Lake Tahoe Lectures, June-July, (1989).

[O3] Q Quantum symmetry, differential geometry of finite graphs and classification of subfactors, University of Tokyo Seminary Notes 45, (Notes recorded by Y. Kawahigashi), 1991.

[O4] _ An invariant coupling between 3-manifolds and subfactors, with connections to topological and conformal quantum field theory, Preprint, (1991).

[P1] Popa, S., Orthogonal pairs of $*$-algebras in finite von Neumann algebras, J. Oper. Theory, 9 (1983), 253-268.

[P2]_, Correspondences, Preprint.

[P3] Classification of subfactors : reduction to commuting squares, Invent. Math., 101 (1990), 19-43.

[P4] Classification of amenable subfactors of type II, Acta Math., 172 (1994), 352-445.

[P5] Classification of actions of discrete amenable groups on amenable subfactors of type II, Preprint, (1992). 
[Pi-Po1] Pimsner, M. and Popa, S., Entropy and index for subfactors, Ann. Scient. Éc. Norm. Sup., 19 (1986), 57-106.

[Pi-Po2] _ Iterating the basic constructions, Trans. Amer. Math. Soc., 310 (1988), 127-134.

[R] Roche, Ph., Ocneanu cell calculus and integrable lattice models, Comm. Math. Phys., 127 (1990), 395-424.

[S] Sekine, Y., Connections associated with finite dimensional Kac algebra actions, Kyushu J. Math., 49 (1995), 253-269.

[Sz] Szymanski, W., Finite index subfactors and Hopf algebra crossed products, Proc. Amer. Math. Soc., 120 (1994), 519-528.

[T] Turaev, V.G., Quantum invariants of knots and 3-manifolds, Stud. Math. 18, Walter de Gruyter, 1994.

[T-V] Turaev, V.G. and Viro, O.Y., State sum invariants of 3-manifolds and quantum 6jsymbols, Topology, 31 (1992), 865-902.

[W] Wenzl, H., Hecke algebras of type $\mathrm{A}_{n}$ and subfactors, Invent. Math., 92 (1988), 345-383.

[Y1] Yamagami, S., A note on Ocneanu's approach to Jones' index theory, Internat. J. Math., 4 (1993), 859-871.

[Y2] On Ocneanu's characterization of crossed products, Preprint, (1993).

[Ya] Yamanouchi, T., Construction of an outer action of a finite-dimensional Kac algebra on the AFD factor of type $\mathrm{II}_{1}$, Internat. J. Math., 4 (1993), 1007-1045. 